\title{
ORDINARY AND STRONG ELLIPTICITY \\ IN THE EQUILIBRIUM THEORY \\ OF INCOMPRESSIBLE HYPERELASTIC SOLIDS
}

Thesis by

Layne Zee

In Partial Fulfillment of the Requirements

for the Degree of

Doctor of Philosophy

California Institute of Technology

Pasadena, California

7983

(Submitted Jury 30, 1982) 
$-i i-$

In Memory of My Father 


\section{Acknowledgments}

My deepest appreciation is extended to Professor E. Sternberg, whose active interest, concern, and advice have had a major influence not only on this dissertation, but on my whole academic experience.

I wish to thank Susan Berkley and Ruth Stratton for their painstaking typing of the manuscript. The financial support supplied by an Earle C. Anthony Fellowship and by the California Institute of Technology, as well as that provided under Contract N00014-75-C-0196 with the Office of Naval Research ir, Washington, D.C., is gratefully acknowledged.

Finally, to Laurie Cook, who sustained and strengthened me throughout the frustrations inherent in completing this work, I owe a debt not easily measured. 
Summary

In this paper explicit necessary and sufficient conditions are established for the ordinary and strong ellipticity of the three-dimensional field equations in the nonlinear equilibrium theory of incompressible, homogeneous and isotropic, hyperelastic solids. The resulting system of inequalities involves the local principal stretches directly and in addition restricts the first and second partial derivatives of the strainenergy density with respect to the deformation invariants or the principal stretches. The conditions of ordinary and strong ellipticity are found to coalesce for materials that obey the Baker-Ericksen inequalities and possess a positive shear modulus at infinitesimal deformations. Various implications of these ellipticity conditions for special classes of materials and deformations are explored. 
Table of Contents

Page

Acknowledgments

Summary iv

Table of Contents $\mathrm{v}$

Introduction 1

$\begin{array}{lll}\text { Sectión } 1 & \text { Preliminaries from finite elastostatics. } & 6\end{array}$ Ordinary and strong ellipticity for incompressible, hyperelastic solids.

Section 2 Explicit necessary conditions for ordinary ellipticity in the case of isotropy.

Section 3 Sufficiency of the conditions necessary for ordinary ellipticity. Explicit necessary and sufficient conditions for strong ellipticity in the case of isotropy.

Section 4 Ellipticity conditions for special classes of deformations and materials.

References

Figures 


\section{Introduction}

Issues related to the ellipticity of the equations governing the finite equilibrium theory of perfectly elastic solids and to the possible breakdown of ellipticity in nonlinear elastostatics, have attracted increasing attention during recent years. Moreover, this interest has sprung from diverse and - to some extent - conflicting motivations.

Thus, some of the work to which we are alluding seeks to extend the scope of the theory to equilibrium solutions of reduced regularity that encompass discontinuous deformation gradients of the kind associated with so-called localized shear failures. The emergence of such singular equilibrium fields in homogeneous hyperelastic solids is accompanied by a failure of ordinary ellipticity. Closely allied in purpose are investigations concerned with bifurcations of equilibrium solutions that are contingent upon a loss of strong ellipticity. In contrast, other related work has a different incentive: it aims chiefly at restrictions of the strain-energy density arising from the postulate of "material stability" in the sense of Hadamard, which precludes a loss of strong ellipticity in the elastostatic field equations.

Although the present paper bears on both of these objectives, it derives its impetus from the first of the foregoing two motivations and continues a sequence of studies initiated in [1]. The latter was prompted by the surmise that a certain crack problem in finite elastostatics of compressible hyperelastic solids fails to admit a solution of unlimited smoothness for a particular hypothetical isotropic material. This conjecture, in turn, suggested that the corresponding displacement equations 
of equilibrium suffer a loss of ordinary ellipticity in the presence of severe enough deformations. That such is indeed the case is borne out by the analysis in [1], which supplies an appropriate necessary and sufficient criterion of ellipticity.

Explicit conditions necessary and sufficient for ordinary and strong ellipticity of the two-dimensional field equations in the theory of finite plane strain for compressible isotropic hyperelastic bodies are deduced in [2]. The results of [2] are applied in [3] to a local study of plane deformation fields that possess continuous displacements, but exhibit finite jump discontinuities in the deformation gradient. The energetics of such "elastostatic shocks" are further explored by Knowles [4].

A necessary and sufficient ellipticity criterion confined to antiplane shear deformations of a class of incompressible isotropic, perfectly elastic materials is included in [4] and applied in [5]. Further, results essentially analogous to those contained in [2], [3], [4], but pertaining to plane deformations of incompressible hyperelastic solids, are derived by Abeyaratne [6], who limits his attention to ordinary ellipticity.

Specific boundary-value problems involving a loss of ellipticity and the concomitant appearance of elastostatic shocks are treated in a number of publications, all but one of which deal asymptotically with the equilibrium field near the tip of a crack in an incompressible body subjected to anti-plane shear. References to these papers can be found in a recent survey $[7] .7$

The present investigation furnishes explicit necessary and sufficient

\footnotetext{
To these we append an asymptotic study by Abeyaratne [8], which has appeared since.
} 
ellipticity conditions appropriate to the general three-dimensional field equations for incompressible isotropic hyperelastic bodies. Further, both ordinary and strong ellipticity are considered, and it is shown that the corresponding ellipticity criteria merge for a material obeying the Baker-Ericksen inequalities, provided its shear modulus is positive at infinitesimal deformations. Thus, in these circumstances, ordinary implies strong ellipticity.

As far as additional related literature is concerned, we cite first an analysis of localized shear failures due to Rudnicki and Rice [9], as well as a bifurcation analysis by Hill and Hutchinson [10]. Both of these papers presuppose a potential loss of ellipticity in equilibrium continuum mechanics; their constitutive settings, however, go beyond purely elastic behavior. Finaliy, it should be emphasized that the work reported here is rather ciosely connected with, and complementary to, a series of investigations due to Sawyers and Rivlin, starting with [11]. These papers are cited in a survey article by Sawyers [12], which summarizes the results obtained. The latter include various necessary conditions for strong ellipticity within the context of the equilibrium theory of incompressible, homogeneous and isotropic, hyperelastic solids. These conditions, which are sufficient merely for certain restricted classes of such materials or when the deformation exhibits a particular degeneracy, are included among the complete ellipticity criteria established in the present paper.

In Section 1, which is partly expository, we assemble some essential ingredients of the nonlinear equilibrium theory for homogeneous incompressible hyperelastic bodies. Here we also define the appropriate concepts of ordinary and strong ellipticity. Proceeding from these two definitions, we establish corresponding necessary and sufficient ellipticity conditions 
that encompass - but are not confined to - the special case of material isotropy treated in the remainder of the paper.

Section 2 is devoted to the derivation of explicit necessary conditions for ordinary ellipticity pertaining to the subclass of isotropic materials. The ensuing inequalities involve the principal stretches directly, as well as through the first and second partial derivatives of the strain-energy density with respect to the deformation invariants. Further, at the end of Section 2, we deduce an equivalent system of inequalities necessary for ordinary ellipticity that depends upon the elastic potential exclusively through its first and second gradients with respect to the principal stretches and is fully symmetric in the latter.

In Section 3 we first establish the sufficiency of the necessary conditions for ordinary ellipticity arrived at in Section 2 . We then derive explicit necessary and sufficient conditions for the strong ellipticity of the elastostatic field equations on the assumption of material isotropy. Finally, we show that the conditions of ordinary and strong ellipticity coalesce in this instance under certain mild and physically plausible additional restrictions of the material response.

The concluding Section 4 deals with applications of the ellipticity conditions established earlier to particular classes of deformations and to special types of elastic materials within the category under consideration. Here we examine the degenerate instances of a locally axisymmetric and a locally plane deformation, as well as the case in which the elastic potential depends merely on a single deformation invariant. Next, we infer the ellipticity, at all deformations, of the equilibrium field equations appropriate to a Mooney-Rivlin material. Finally, as an illustrative example, we discuss in detail the domain of ellipticity in the 
space of the principal stretches for a specific material that has an elastic potential of a form proposed by Ogden [13]. 
1. Preliminaries from finite elastostatics. Ordinary and strong ellipticity for imcompressible, hyperelastic solids.

In this section we recall certain prerequisites from the finite equilibrium theory of homogeneous incompressible hyperelastic solids. We then define the notions of ordinary and strong ellipticity in the present context and deduce necessary and sufficient ellipticity conditions that are not contingent upon any material symmetry restrictions.

Throughout this paper, uppercase boldface letters denote second-order tensors as well as three-by-three matrices; lowercase boldface letters denote vectors and also three-rowed column matrices. Further, the same boldface letter will be used to designate a tensor or vector and its matrix of scalar components in the underlying rectangular Cartesian coordinate frame.

Let $Q$ be the three-dimensional open region occupied by the interior of a body in an undeformed reference configuration. A deformation of the body is then described by a transformation

$$
y=\underset{\sim}{\hat{y}}(x)=\underset{\sim}{x}+\underset{\sim}{u}(x) \text { on } R \text {, }
$$

which maps $Q$ onto a domain $a_{\star}$. Here $\underset{\sim}{x}$ is the position vector of a generic point in $R, \underset{\sim}{\hat{y}}(x)$ is its deformation image in $R_{*}$, and $\underset{\sim}{u}$ is the displacement vector field. Thus, $x_{i}$ and $y_{i}$ are the Cartesian material and spatial coordinates, respectively. We shall suppose for the time being that the mapping $\underset{\sim}{\hat{\sim}}$ is twice continuously differentiable and

\footnotetext{
Latin subscripts have the range $(1,2,3)$ and summation over repeated subscripts is taken for granted.
} 
one-to-one on $R$. Next, let

$$
\underset{\sim}{F}=\nabla \underset{\sim}{\hat{y}}=\left[\partial \hat{y}_{i} / \partial x_{j}\right]=\left[\delta_{i j}+\partial u_{i} / \partial x_{j}\right], \quad J=\operatorname{det} \underset{\sim}{F} \text { on } R,
$$

so that $\underset{\sim}{F}$ is the deformation-gradient tensor and $J$ the Jacobian deter-

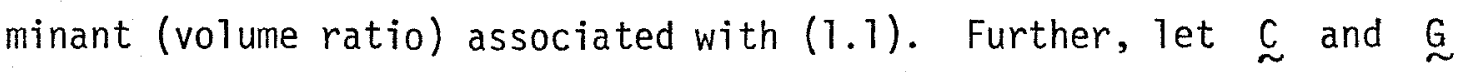
stand for the right and left Cauchy-Green deformation tensors, whence

$$
\underset{\sim}{C} \underset{\sim}{F_{\sim}^{\top}} \underset{\sim}{F}, \underset{\sim}{G}=\underset{\sim}{F} F^{\top} \text { on } R
$$

Both $\underset{\sim}{\mathcal{C}}$ and $\underset{\sim}{G}$ are symmetric, positive-definite tensors, which have the same fundamental scalar invariants $I_{i}$ and hence possess common positive principal values $\lambda_{j}^{2}$; consequently, $\lambda_{i}>0$ are the principal stretches of the deformation at hand. Thus,

$$
\begin{aligned}
& I_{1}=\operatorname{tr} \underset{\sim}{\mathcal{C}}=\lambda_{1}^{2}+\lambda_{2}^{2}+\lambda_{3}^{2},
\end{aligned}
$$

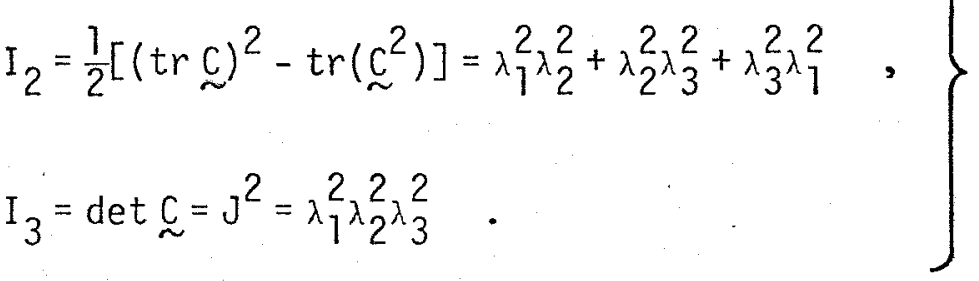

Since the material is assumed to be incompressible, only locally volume-preserving deformations are admissible. Therefore,

$$
J=\lambda_{1} \lambda_{2} \lambda_{3}=1, \quad I_{3}=1 \text { on } R
$$

Let $\underset{\sim}{\sim}$, defined on $\Omega$, be the nominal (Piola) stress-tensor field

\footnotetext{
If $M$ is a three-by-three matrix with elements $M_{i j}$, we alternatively write $^{\sim}\left[M_{i j}\right]$ in place of $\underset{\sim}{M} ; \delta_{i j}$ is the Kronecker-delta. ${ }^{2}$ A superscript $T$ will always indicate transposition.
} 
accompanying the deformation. The equilibrium balance of linear and angular momentum, in the absence of body forces, then demands that

$$
\operatorname{div} \underset{\sim}{\sigma}=\underset{\sim}{\sim}, \underset{\sim}{\sigma} \underset{\sim}{F^{\top}}=\underset{\sim}{F} \underset{\sim}{\sigma} \text { or } \sigma_{i j, j}=0, \sigma_{i k} F_{j k}=F_{i \ell} \sigma_{j \ell} \text { on } R . \quad(1.6)^{1}
$$

Further, suppose $\underset{\sim}{\sim}$ is the true (Cauchy) stress-tensor field on $R_{*}$. Accordingly,

$$
\underset{\sim}{\tau}(\underset{\sim}{\hat{y}}(x))=\underset{\sim}{\sigma} \underset{\sim}{(x)} \underset{\sim}{F^{\top}}(\underset{\sim}{x}) \forall \underset{\sim}{x} \in R
$$

Turning now to the governing constitutive relations, we call $W$ the elastic potential of the hyperelastic material under consideration. The scalar-valued response function $w$, which represents the strain-energy density per unit undeformed volume, is taken to be defined and at least twice continuously differentiable on the set $\mathcal{L}$ of all nonsingular secondorder tensors. The appropriate constitutive law may then be written as

$$
\underset{\sim}{\sigma}=W_{\sim}(\underset{\sim}{F})-p F_{\sim}^{-T} \text { or } \sigma_{i j}=\partial W(\underset{\sim}{F}) / \partial F_{i j}-p F_{j i}^{-1} \text {, }
$$

in which $p$, for the present assumed to be continuously differentiable on $R$, stands for the arbitrary pressure field needed to accommodate the constraint of incompressibility (1.5). ${ }^{3}$

The elastic potential $W$ is subject to the requirement of material

TSubscripts preceded by a comma indicate partial differentiation with respect to the corresponding material Cartesian coordinate.

${ }^{2}$ Here and in the sequel, $\sim^{-T}$ denotes the transposed inverse of the tensor $\underset{\sim}{\mathrm{F}}$, while $\mathrm{F}_{j i}^{-1}=\left(\mathrm{F}^{-\mathrm{T}}\right)_{i j}$.

3 We emphasize that although this constraint restricts the argument of $W(F)$ to unimodular tensors, the particular manner in which the domain of definition of $W$ is extended to the set $\mathcal{L}$ of all nonsingular tensors affects merely the pressure $p$, and is therefore irrelevant. 
frame indifference, which demands that

$$
W(\underset{\sim}{F})=W(\underset{\sim}{\mathbb{N}}) \forall(\underset{\sim}{Q}, F) \in \sigma \times £
$$

where $\theta$ is the set of all proper orthogonal second-order tensors. Moreover, (1.8) and (1.9) are found to imply the second of (1.6) and hence the symmetry of the true stress-tensor field $\underset{\sim}{\sim}$.

Substituting from (1.8) into the first of (1.6), appealing to (1.2), and recalling that $F_{j i, j}^{-1}=0$ for a unimodular deformation-gradient field, one is led to the displacement-equations of equilibrium. Adjoining to the latter the incompressibility condition (1.5), one arrives at the system of partial differential equations

$$
c_{i j k \ell}(F) u_{k, \ell j}-p, j F_{j i}^{-1}=0, j=\operatorname{det} \underset{\sim}{F}=1 \text { on } \Omega,
$$

provided $c_{i j k q}(E)$ are the components of the fourth-order tensor defined by

$$
c_{i j k \ell}(E)=c_{k \ell i j}(\underset{\sim}{F})=a^{2} W(\underset{\sim}{F}) / \partial F_{i j} \partial F_{k \ell} .
$$

In the case of material isotropy, the strain-energy density $W(F)$ involves $\underset{\sim}{\mathrm{F}}$ only through the invariants $\mathrm{I}_{1}$ and $\mathrm{I}_{2}$ of $\underset{\sim}{\mathrm{C}}=\underset{\sim}{\mathrm{F}^{\top}} \underset{\sim}{\mathrm{F}}$. Thus,

$$
W(E)=\hat{W}\left(I_{7}(\underset{\sim}{F}), I_{2}(\underset{\sim}{F})\right) .
$$

From (1.3) and (1.4) follow

$$
\frac{\partial I_{1}}{\partial F_{i j}}=2 F_{i j}, \frac{\partial I_{2}}{\partial F_{i j}}=2 I_{1} F_{i j}-2 G_{i \ell \ell j} F_{l j}, \frac{\partial J}{\partial F_{i j}}=J F_{j i}^{-1} .
$$

For future convenience we adopt the abridged notation 


$$
\hat{W}_{\alpha}=\partial \hat{W}\left(I_{1}, I_{2}\right) / \partial I_{\alpha}, \hat{W}_{\alpha \beta}=\partial^{2} \hat{W}^{-10}\left(I_{1}, I_{2}\right) / \partial I_{\alpha} \partial I_{\beta} .
$$

Equations $(1.8),(1.12),(1.13),(1.14)$ then yield the stress-deformation relations for isotropic incompressible hyperelastic solids in the form

$$
\left.\begin{array}{l}
\sigma_{i j}=2\left(\hat{W}_{1}+I_{1} \hat{W}_{2}\right) F_{i j}-2 \hat{W}_{2} G_{i \ell} F_{\ell j}-p F_{j j}^{-1} \\
\stackrel{\sigma}{\sigma}=2\left(\hat{W}_{1}+I_{1} \hat{W}_{2}\right) \underset{\sim}{ }-2 \hat{W}_{2} \underset{\sim}{G}-p F_{\sim}^{-T} .
\end{array}\right\}
$$

On the other hand, on account of (1.3), (1.7), (1.15), the true stress field $\underset{\sim}{\sim}$ obeys the constitutive relation.

$$
I=2\left(\hat{W}_{1}+I_{1} \hat{W}_{2}\right) G-2 \hat{W}_{2} G^{2}-p I
$$

where 1 stands for the idem tensor with the components $\delta_{i j}$.

According to (1.16), the principal axes of $\underset{\sim}{\tau}$ and $\underset{\sim}{G}$ coincide;

further, if $\tau_{i}$ designates the principal true stress associated with the principal stretch $\lambda_{j}$, one evidently has

$$
\tau_{i}=-p+2 \lambda_{i}^{2}\left[\hat{W}_{1}+\left(I_{1}-\lambda_{i}^{2}\right) \hat{W}_{2}\right] \text { (no sum). }
$$

Later on, we shall need to refer to the Baker-Ericksen inequalities, which require that

$$
\left(\tau_{i}-\tau_{j}\right)\left(\lambda_{i}-\lambda_{j}\right)>0 \text { if } \lambda_{i} \neq \lambda_{j} \text { (no sum), }
$$

and thus postulate that the greater true principal stress occurs always in the direction of the larger principal stretch. Because of (1.17) and (1.5), the inequality (1.18) is equivalent to

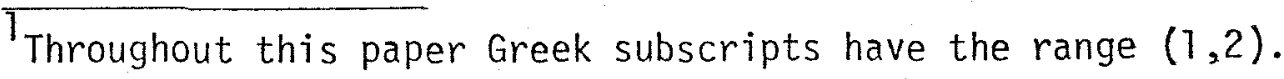




$$
\hat{W}_{1}+\left(\lambda_{i} \lambda_{j}\right)^{-2} \hat{W}_{2}>0 \text { if } \lambda_{i} \neq \lambda_{j}
$$

With a view toward defining the notion of ordinary ellipticity relevant to the system of partial differential equations $(1.10)^{1}$, we consider a surface $\&$ lying wholly within $\Omega$ that admits a twice continuously differentiable and one-to-one parameterization

$$
\underset{\sim}{x}=\underset{\sim}{\sim}\left(\xi_{1}, \xi_{2}\right) \forall\left(\xi_{1}, \xi_{2}\right) \in \Sigma,
$$

where $\Sigma$ is a region of the parameter-plane and $\left(\xi_{7}, \xi_{2}\right)$ are orthogonal curvilinear coordinates on $\mathcal{S}$. Let $P$ be an arbitrarily chosen, fixed point on $\mathcal{H}$. Then, within a three-dimensional neighborhood of $P$, we may introduce orthogonal curvilinear coordinates $\left\langle\xi_{1}, \xi_{2}, \zeta\right)$, such that

$$
\underset{\sim}{x}=\underset{\sim}{x}\left(\xi_{1}, \xi_{2}, \zeta\right)=\stackrel{*}{\underset{\sim}{x}}\left(\xi_{1}, \xi_{2}\right)+\zeta \underset{\sim}{n}\left(\xi_{1}, \xi_{2}\right),
$$

in which $\underset{\sim}{n}$ is the unit normal vector of $\mathscr{S}$ and $|\zeta|$ is the perpendicular distance from $\mathscr{f}$ of a point with position vector $\underset{\sim}{x}$ The mapping (1.21) is locally one-to-one; let its inverse be given by

$$
\xi_{\alpha}=\hat{\xi}_{\alpha}(\underset{\sim}{x}), \quad \zeta=\hat{\zeta}(\underset{\sim}{x})
$$

where $\hat{\xi}_{\alpha}$ and $\hat{\zeta}$ are defined and twice continuously differentiable in a neighborhood of $P$.

We now weaken the original smoothness requirements on $\underset{\sim}{u}$ and $p$. Thus, we assume that $(\underset{\sim}{u}, p)$ is a solution of (1.10) with $\underset{\sim}{u}$ continuousiy differentiable and merely piecewise twice continuously differentiable and

What follows is at once an adaptation to incompressible bodies of the analysis in Section 1 of [1] and a generalization to three dimensions of the development in Section 3.1 of [6]. 
p continuous but only piecewise continuously differentiable on $\Omega$. We shall call such a $(\underset{\sim}{u}, p)$ a "relaxed solution" of (1.10). On setting

$$
\left.\underset{\sim}{\bar{u}}\left(\xi_{1}, \xi_{2}, \zeta\right)=\underset{\sim}{u} \underset{\sim}{\operatorname{x}}\left(\xi_{1}, \xi_{2}, \zeta\right)\right), \bar{p}\left(\xi_{1}, \xi_{2}, \zeta\right)=p\left(\bar{\sim}\left(\xi_{1}, \xi_{2}, \zeta\right)\right),
$$

one confirms with the aid of (1.22) that

$$
\begin{aligned}
& \left., u_{k, \ell j}=\frac{\partial \bar{u}_{k}}{\partial \xi_{\alpha}} \hat{\xi}_{\alpha, \ell j}+\frac{\partial \bar{u}_{k}}{\partial \zeta} \hat{\zeta}_{, \ell j}+\frac{\partial^{2} \bar{u}_{k}}{\partial \xi_{\beta} \partial \xi_{\alpha}} \hat{\xi}_{\alpha, \ell} \hat{\xi}_{k, j}+\frac{\partial^{2} \bar{u}_{k}}{\partial \zeta \partial \xi_{\alpha}} \hat{\xi}_{\alpha, l} \hat{\zeta}_{, j}\right] \\
& +\frac{\partial^{2} \bar{u}_{k}}{\partial \xi_{\alpha} \partial \zeta} \hat{\zeta}, \ell^{\hat{\xi}_{\alpha, j}}+\frac{\partial^{2} \bar{u}_{k}}{\partial \zeta^{2}} \hat{\zeta}, \ell^{\hat{\zeta}, j} \\
& p_{, j}=\frac{\partial \bar{p}}{\partial \xi_{\alpha}} \hat{\xi}_{a, j}+\frac{\partial \bar{p}}{\partial \zeta} \hat{\zeta}_{, j} \cdot
\end{aligned}
$$

On account of the assumed smoothness of $\underset{\sim}{u}, p$ and of the mapping (1.21), the first and second-order partial derivatives of $\bar{u}_{k}$ and the first-order partial derivatives of $\bar{p}$, except possibly $\partial^{2} \bar{u}_{k} / \partial \zeta^{2}$ and $\partial \bar{p} / \partial \zeta$, are continuous in the neighborhood of $P$ under consideration; further, the latter two derivatives may at most have finite jump discontinuities across \&. Mareover, (1.24) and the smoothness of the inverse mapping (1.22) give

$$
\llbracket u_{k, \ell j} \rrbracket=\llbracket \partial^{2} \bar{u}_{k} / \partial \zeta^{2} \rrbracket \hat{\zeta}_{,}, \hat{\zeta}_{, j}, \llbracket p, j \rrbracket=\llbracket \partial \bar{p} / \partial \zeta \rrbracket \hat{\zeta}, j \text { on } \mathcal{\&} \text {, }
$$

where [h] denotes the jump of a function $h$ across $\mathcal{S}$.

The second of (1.10) implies $J_{, j}=0$, which - because of (1.2), (1.5), and the last of $(1.13)$ - is equivalent to

$$
F_{l k}^{-1} u_{k, \ell j}=0 \text { on } \Omega
$$


Consequently, in view of (1.25), (1.26) and the continuity of $\underset{\sim}{F}$ and of $c_{i j k \ell}$, equations $(1.10)$ yield

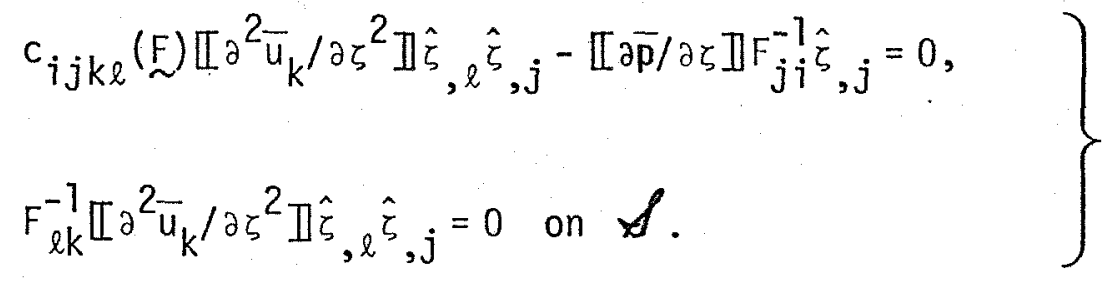

Also, noting that $\nabla \hat{\zeta} /|\nabla \hat{\zeta}|$ on $\&$ coincides with the unit normal vector $\stackrel{n}{\sim}$ of $\mathscr{f}$ and defining

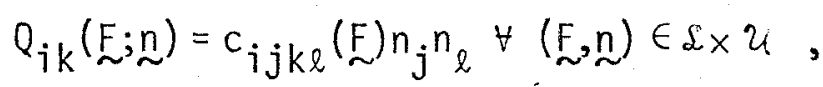

$$
\begin{aligned}
& v=\llbracket \partial^{2} \bar{u} / \partial \zeta^{2} \rrbracket, q=|\nabla \hat{\zeta}|^{-1} \llbracket \partial \bar{p} / \partial \zeta \rrbracket \text { on } \mathcal{A} \text {, }
\end{aligned}
$$

where $u$ is the set of all unit vectors, we infer that

$$
Q(F ; \underset{\sim}{n}) \underset{\sim}{\sim}-q F_{\sim}^{-T} \underset{\sim}{n}=\underset{\sim}{0}, \underset{\sim}{v} \cdot\left(F_{\sim}^{-T} \underset{\sim}{n}\right)=0 \text { on } \mathcal{S}
$$

Clearly, (1.29) constitute four linear homogeneous algebraic equations in the jumps $\left(v_{j}, q\right)$, which admit oniy the trivial solution $v_{i}=0, q=0$ if and only if the determinant of their coefficientmatrix fails to vanish.

The system (1.10) is said to be elliptic at a relaxed solution $(\underset{\sim}{u}, p)$ and at a point $x \in R$ if and only if $\underset{\sim}{u}$ is twice continuously differentiable and $p$ is continuously differentiable at $\underset{\sim}{x}$. Accordingly, when (1.10) is elliptic at $(\underset{\sim}{u}, p)$ and $\underset{\sim}{x}$, there does not exist any surface (of the requisite smoothness) through the point $\underset{\sim}{x}$ across which the "second normal derivative" $\partial^{2} \bar{u} / \partial \zeta^{2}$ or the "normal derivative" $\partial \bar{p} / \partial \zeta$ is discontinuous.

It is evident from (1.29) that (1.10) is elliptic at a relaxed solution There $Q_{i k}$ are the components of the acoustic tensor $\underset{\sim}{Q}$, which is symmetric
because of $(1.11)$. 
$(\underset{\sim}{u}, p)$ and at a point $\underset{\sim}{x}$ if and only if

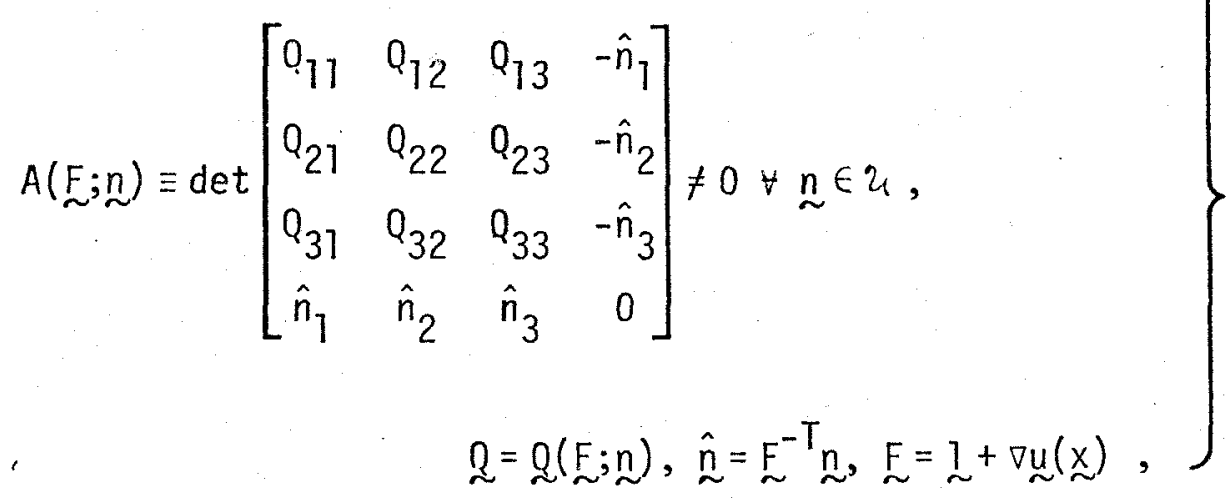

or equivalently

$$
\left.\begin{array}{c}
A(\underset{\sim}{F ; n})=\frac{1}{2} \varepsilon_{i j k} \varepsilon_{\ell m n} \hat{n}_{i} \hat{n}_{\ell} Q_{j m} 0_{k n} \neq 0 \quad \forall \underset{\sim}{n} \in U, \\
Q=Q \underset{\sim}{Q}(\underset{\sim}{F} \underset{\sim}{n}), \underset{\sim}{\hat{n}}=F_{\sim}^{-T} \underset{\sim}{n}, \underset{\sim}{F}=\underset{\sim}{q}+\underset{\sim}{u}(\underset{\sim}{x}),
\end{array}\right\}
$$

where $\varepsilon_{i j k}$ stands for the components of the three-dimensional alternator. If (1.31) is violated for some unit vector $\underset{\sim}{n}$, then $\underset{\sim}{n}$ is normal to a material characteristic surface in $\Omega$ through $\underset{\sim}{x}$. These characteristic surfaces are the only possible carriers of discontinuities in $\partial^{2} \bar{u} / \partial \zeta^{2}$ or $\partial \bar{p} / \partial \zeta$, and ordinary ellipticity precludes the existence of real characteristic surfaces.

We turn next to the definition of strong ellipticity ${ }^{1}$ appropriate to the system of partial differential equations (1.10). For this purpose we consider an infinitesimal plane displacement-pressure wave superposed upon a finite homogeneous deformation of an incompressible hyperelastic body occupying the entire three-dimensional space $\varepsilon$. Thus, if $t$ denotes

This concept is essentially identical with the notion of Hadamard stability. What follows is parallel to the analysis in Section 3 of [12], which is however confined to isotropic materials. 
the time, while $\stackrel{\circ}{\underset{\sim}{\sim}}$ and $\stackrel{\circ}{\mathrm{p}}$ designate the constant deformation-gradient and pressure fields associated with the homogeneous pre-deformation, we set

$$
\underset{\sim}{\hat{y}}(\underset{\sim}{x}, t)=\underset{\sim}{\stackrel{0}{x}} \underset{\sim}{\sim}+\underset{\sim}{w}(\underset{\sim}{x}, t), p(\underset{\sim}{x}, t)=\stackrel{\circ}{p}+r(\underset{\sim}{x}, t) \forall(\underset{\sim}{x}, t) \in \varepsilon \times(-\infty, \infty),
$$

regard $|\nabla w|$, as well as $|r|$, small compared to unity, and take

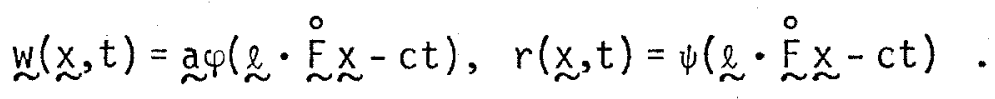

Here $\underset{\sim}{a}$ and $\stackrel{\&}{\sim}$ are constant unit vectors, determining the direction of motion and the direction of propagation of the plane wave at hand, whereas $c \neq 0$ is its speed of propagation. Further, $\varphi$ and $\psi$ are scalar-valued functions, the first of which is twice - the second once - continuously differentiable on $(-\infty, \infty)$. Finally, we take for granted that the derivatives $\varphi^{\prime \prime}$ and $\psi^{\prime}$ fail to vanish identically. From (1.32) and the incompressibility requirement, one has

$$
\underset{\sim}{F}=\nabla \underset{\sim}{\hat{y}}=\stackrel{\circ}{F}+\nabla \underset{\sim}{W}, J=\operatorname{det} \underset{\sim}{F}=1 \text { on } \mathcal{E} \times(-\infty, \infty), \operatorname{det} \underset{\sim}{\stackrel{F}{F}}=1 .
$$

The time-dependent nominal stresses induced by the motion (1.32), (1.33) follow from the constitutive relation (1.8) and are now subject to the stress equations of motion

$$
\sigma_{i j, j}=\rho \partial^{2} u_{i} / \partial t^{2} \text { on } \varepsilon \times(-\infty, \infty) \text {, }
$$

provided $\rho>0$ is the constant mass density and $\underset{\sim}{u}$ is the displacement field. In view of the first of (1.32), we see that

$$
u_{i}(\underset{\sim}{x}, t)=\hat{y}_{j}(\underset{\sim}{x}, t)-x_{i}=\left(\stackrel{0}{F}_{i j}-\delta_{i j}\right) x_{j}+w_{i}(\underset{\sim}{x}, t)
$$

Recalling the identities 


$$
\operatorname{det} \underset{\sim}{F}=\frac{1}{6} \varepsilon_{i j k^{\varepsilon}}{ }_{p q r} F_{i p} F_{j q} F_{k r},(\operatorname{det} E) F_{j i}^{-1}=\frac{1}{2} \varepsilon_{i k \ell} \varepsilon_{j p q} F_{k p} F_{\ell q} \forall \underset{\sim}{E} \in \mathcal{E} \text {, }
$$

we substitute from (1.34) into (1.37) and, upon a linearization with respect to $\underset{\sim}{\sim}$, arrive at

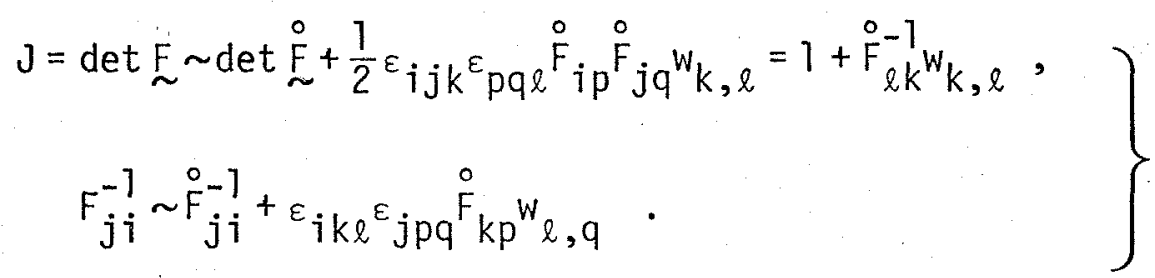

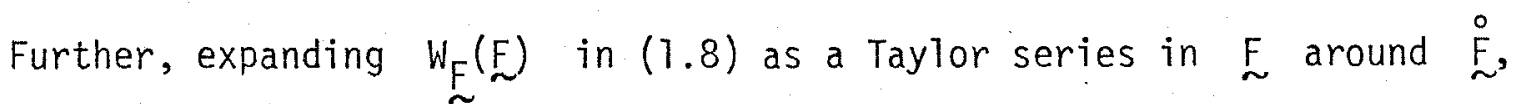
appealing to (1.11), (1.34), as well as (1.32), (1.38), and linearizing with respect to $\nabla \underset{\sim}{w}$ and $r$, we obtain

$$
\left.\sigma_{i j} \sim \frac{\partial W(E)}{\partial F_{i j}}\right|_{E=\stackrel{\circ}{F}}-\stackrel{\circ}{p}_{j}^{\circ} F_{j i}^{-1}+c_{i j k \ell} \stackrel{\circ}{\stackrel{F}{F}) W_{k, l}}-\stackrel{\circ}{p} \varepsilon_{i k \ell} \varepsilon_{j p q} \stackrel{\circ}{F}_{k p} W_{l, q}-r \stackrel{\circ}{F}_{j i}^{-1} .
$$

Now, (1.35) and the second of (1.34), together with (1.39), (1.36), and the first of (1.38), lead to the linearized version of the displacementequations of motion and the linearized incompressibility condition

$$
c_{i j k \ell}(\stackrel{\circ}{\sim}) w_{k, l j}-r, \stackrel{\circ}{F}_{j i}^{-1}=\rho \partial^{2} w_{i} / \partial t^{2}, \stackrel{\circ}{F}_{l k}^{-1} W_{k, l}=0 \text { on } \varepsilon \times(-\infty, \infty) \text {. }
$$

For the plane wave characterized by (1.33), equations (1.40) give

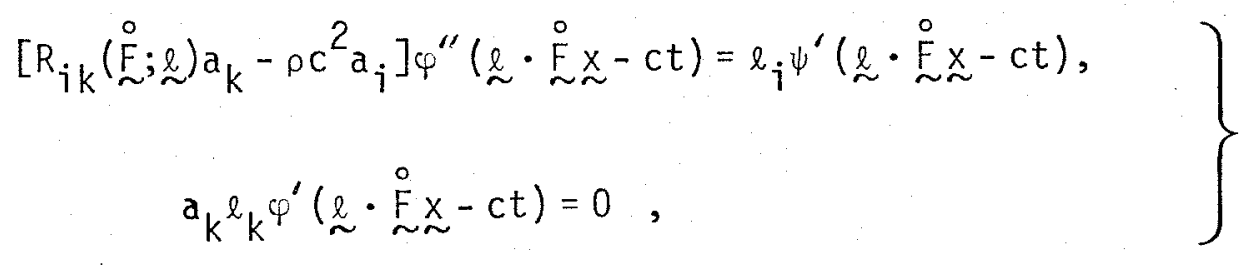

in which

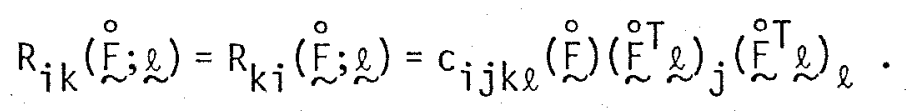


From (1.41) follows

$$
a_{k} k_{k}=0, \psi^{\prime}(\underset{\sim}{\ell} \cdot \underset{\sim}{\stackrel{\circ}{F}} \underset{\sim}{x}-c t)=b \varphi^{\prime \prime}(\underset{\sim}{\ell} \cdot \stackrel{\circ}{\underset{\sim}{F}} x-c t), \quad b=\ell_{i} a_{k} R_{i k}(\stackrel{\circ}{F} ; \ell),
$$

and hence,

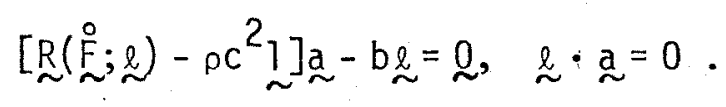

The system (1.44) constitutes four 7 inear homogeneous algebraic equations in $\left(a_{\hat{j}}, b\right)$ and has a nontrivial solution if and only if the determinant of its coefficient matrix equals zero. It is easily confirmed that this determinant may be written as

$$
\begin{aligned}
& \operatorname{det}\left[\begin{array}{cccc}
R_{11}-\rho c^{2} & R_{12} & R_{13} & -\ell_{1} \\
R_{21} & R_{22^{-}} \rho c^{2} & R_{23} & -\ell_{2} \\
R_{31} & R_{32} & R_{33}-\rho c^{2} & -\ell_{3} \\
\ell_{1} & \ell_{2} & l_{3} & 0
\end{array}\right]= \\
& \frac{1}{2} \varepsilon_{i j k} \varepsilon_{p q r_{i} l_{p}}\left(R_{j q}-\rho c^{2} \delta_{j q}\right)\left(R_{k r}-\rho c^{2} \delta_{k r}\right) .
\end{aligned}
$$

On expanding the right-hand side of (1.45), one sees that equations (1.44) have a nontrivial solution $\left(a_{j}, b\right)$ if and only if $\rho c^{2}$ and the unit vector \& satisfy the secular equation

$$
\rho^{2} c^{4}-\left(R_{k k}-R_{i j} i_{i}{ }_{j}\right) \rho c^{2}+\frac{1}{2} \varepsilon_{i j k}{ }^{\varepsilon} p q r^{\ell} i^{\ell} p^{R} j q^{R} k r=0, \quad \underset{\sim}{R}=\underset{\sim}{R}(\underset{\sim}{\stackrel{0}{F} ; \ell}),
$$

which is a quadratic equation in $\rho c^{2}$.

\footnotetext{
Upon specialization to an isotropic material and to a pure homogeneous pre-deformation, (1.46) reduces to equation (3.12) of Sawyers [12], who sets $\sigma=\left(\rho c^{2}\right)^{-1}$.
} 
We show next that the discriminant of (1.46),

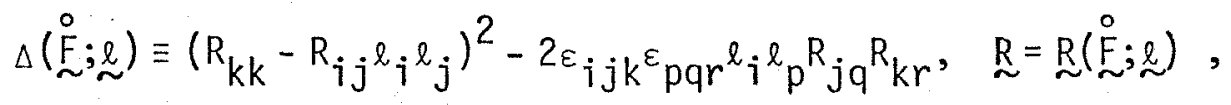

is non-negative for every nonsingular $\underset{\sim}{\stackrel{\circ}{\sim}}$ and every unit vector $\stackrel{\sim}{\sim}$. To this end let

$$
\begin{gathered}
\alpha_{1}=R_{22}-R_{33}, \alpha_{2}=R_{33}-R_{11}, \alpha_{3}=R_{11}-R_{22}, \\
\gamma_{1}=2 R_{23}, \gamma_{2}=2 R_{31}, \gamma_{3}=2 R_{12},
\end{gathered}
$$

and note first that if $\ell_{1}=\ell_{3}=0$, one has $\Delta(\stackrel{\circ}{\underset{\sim}{\sim} ;} \underset{\sim}{\sim})=\alpha_{2}^{2}+r_{2}^{2} \geq 0$. On the other hand, if $\ell_{1}^{2}+\ell_{3}^{2}>0$, one verifies after a straightforward computation making use of $2_{i} l_{i}=1$, that

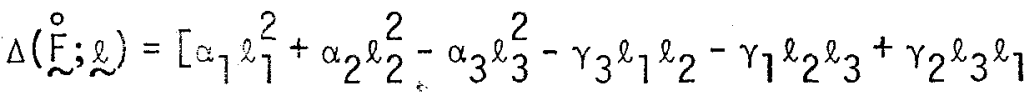

$$
\begin{aligned}
& \left.+2\left(\ell_{1}^{2}+e_{3}^{2}\right)^{-1}\left(r_{2} \ell_{3}-\alpha_{2} \ell_{1}\right) \ell_{1} \ell_{2}^{2}\right]^{2} \\
& +\left[\gamma_{1} \ell_{1}-\gamma_{2} \ell_{2}-\gamma_{3} \ell_{3}+2\left(\ell_{1}^{2}+\ell_{3}^{2}\right)^{-1}\left(\gamma_{2} \ell_{3}-\alpha_{2} l_{1}\right) \ell_{2} \ell_{3}\right]^{2} \geq 0 .
\end{aligned}
$$

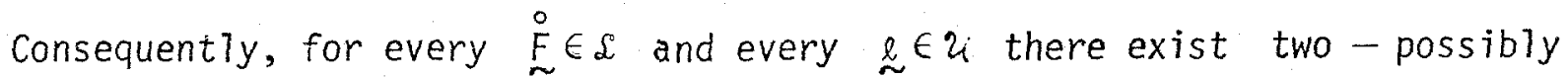
coalescent - real values of $\rho c^{2}$ satisfying the secular equation (1.46).

We proceed now to the relevant definition of strong ellipticity.

The system (1.10) is said to be strongly elliptic at a solution $(u, p)$ and at a point $\underset{\sim}{x} \in R$ if and only if a body of the same material occupying $\mathcal{E}$, having been subjected to a homogeneous deformation with the deformationgradient $\underset{\sim}{\stackrel{\circ}{F}}=\underset{\sim}{1}+\underset{\sim}{\sim}(x)$ and to a constant pressure $\stackrel{\circ}{p}=p(\underset{\sim}{x})$, admits only infinitesimal plane displacement-pressure waves of the form (1.33) with real non-zero propagation speeds. Unless (1.10) is strongly elliptic at $(\underset{\sim}{u}, p)$ 
and $\underset{\sim}{x}$, there is an $\underset{\sim}{\&}$ such that (1.46) is satisfied for two conjugate imaginary values of $c$ or for $c=0$. In the first instance, there exist superposed infinitesimal motions of the form (1.33) with

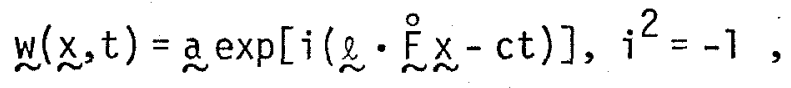

which grow unbounded in time. When $c=0$, in turn, one can satisfy the linearized equations of motion with

$$
\underset{\sim}{w}(\underset{\sim}{x}, t)=\underset{\sim}{a} t \exp (i \underset{\sim}{\ell} \cdot \underset{\sim}{F} \underset{\sim}{x})
$$

so that in either event the body is dynamically unstable.

Evidently, necessary and sufficient in order that (1.10) be strongly elliptic at a solution $(\underset{\sim}{u}, p)$ and at a point $\underset{\sim}{\sim}$ is that both real roots $\rho c^{2}$ of the secular equation $(1.46)$ be positive for $\stackrel{\circ}{\mathcal{F}}=\underset{\sim}{1}+\nabla \underset{\sim}{\operatorname{U}}(\mathrm{x})$ and for every unit vector $\approx$. According to (1.46), this is the case if and only if

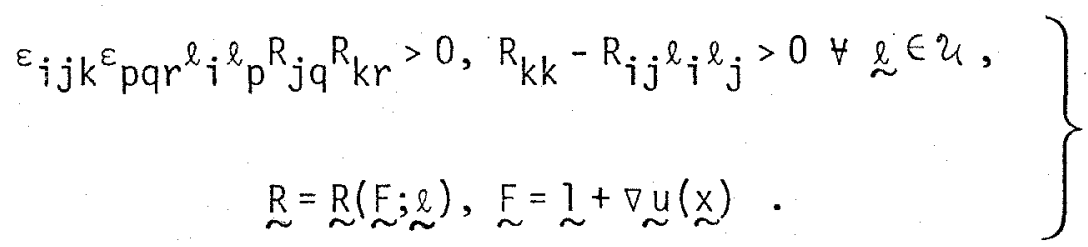

But, from (1.28) and (1.42),

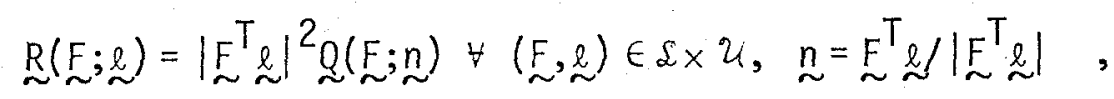

so that the strong-ellipticity conditions (1.47) may be expressed in terms of the acoustic tensor $\underset{\sim}{\sim}$ as

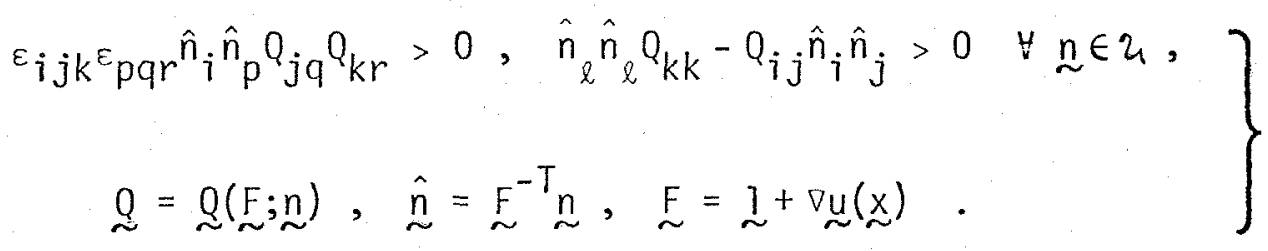


Final1y, by comparing (1.31) with the first of (1.49), we confirm that strong ellipticity implies ordinary ellipticity.

It is essential to remark that conditions (1.31), as well as (1.49), are inadequate as a means for testing the local ellipticity of a particular equilibrium solution appropriate to a specific material within the class under consideration. For the important subclass of isotropic materials, however, one may deduce from (1.31) and (1.49), corresponding intrinsic ellipticity criteria in a form suitable for this purpose. It is this task that constitutes our main goal and to which we presently turn our attention. 
2. Explicit necessary conditions for ordinary ellipticity in the case of isotropy.

Our current objective is to deduce from (1.31), which is necessary and sufficient for ordinary ellipticity, a set of explicit necessary ellipticity conditions for the special case of an isotropic material. This set of conditions involves exclusively the local principal stretches, which enter directly, as well as through the first and second partial derivatives of the strain-energy density with respect to the deformation invariants or the principal stretches.

As a first step, we derive an ellipticity condition equivalent to (1.31), but analytically more amenab]e. ${ }^{1}$ since $\left[\mathrm{C}_{\mathbf{i j}}\right]=\mathrm{F}_{\sim}^{T_{F}}$ is a symmetric matrix with the principal values $\lambda_{j}^{2}$, there is an orthogonal matrix $\underset{\sim}{M}=\underset{\sim}{M}(F)$, such that

$$
\underset{\sim}{M^{\top}} \underset{\sim}{C M}=\underset{\sim}{D}, \quad D_{i j}=\lambda_{i}^{2} \delta_{i j} \quad \text { (no sum). }
$$

Next, for every nonsingular $\underset{\sim}{F}$ and every unit vector $\underset{\sim}{m}$, let $\underset{\sim}{N}=\underset{\sim}{N}(F)$ and $\underset{\sim}{H}=\underset{\sim}{H}(\underset{\sim}{\sim} ; m)$ be the auxiliary matrices defined by

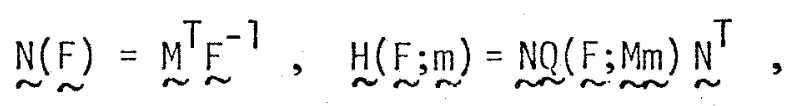

in which $\underset{\sim}{Q}$ is the component matrix of the acoustic tensor introduced in (1.28). Evidently $\underset{\sim}{\mathrm{H}}$ is symmetric. On setting

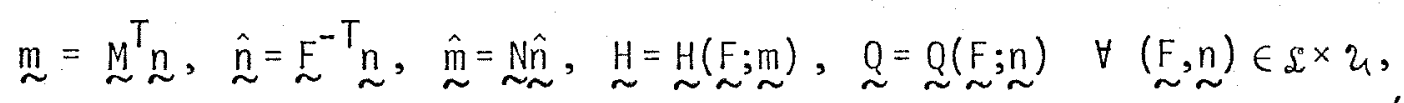

\footnotetext{
What follows is suggested by the development in section 3 of [1].
} 
one readily confirms the block-matrix equation

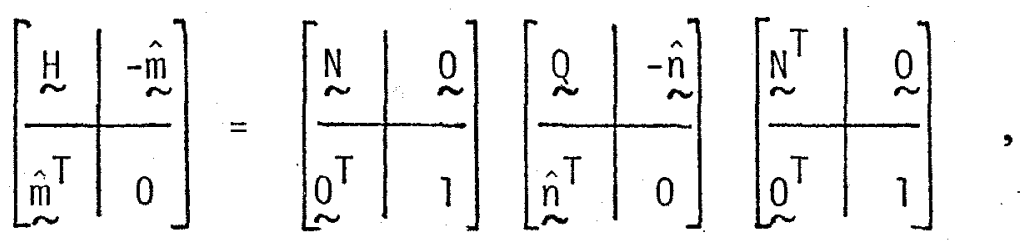

provided $\underset{\sim}{0}$ stands for the null three-rowed column matrix. It follows at once that

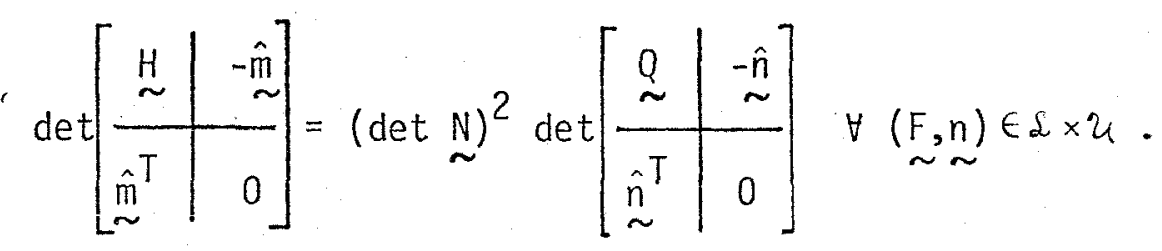

Appealing to (1.30) and (2.1) through (2.4), one sees after elementary manipulations that for every unimodular $\underset{\sim}{\mathrm{F}}$ and every unit vector $\underset{\sim}{\sim}$,

$$
A\left(\underset{\sim}{\mathrm{F} ; \mathrm{m})}=\operatorname{det}\left[\begin{array}{cccc}
\mathrm{H}_{11} & \mathrm{H}_{12} & \mathrm{H}_{13} & -\hat{\mathrm{m}}_{1} \\
\mathrm{H}_{21} & \mathrm{H}_{22} & \mathrm{H}_{23} & -\hat{\mathrm{m}}_{2} \\
\mathrm{H}_{31} & \mathrm{H}_{32} & \mathrm{H}_{33} & -\hat{\mathrm{m}}_{3} \\
\hat{\mathrm{m}}_{1} & \hat{\mathrm{m}}_{2} & \hat{\mathrm{m}}_{3} & 0
\end{array}\right], \quad \hat{\mathrm{m}}=\mathrm{D}_{\sim}^{-1} \stackrel{\sim}{\sim},\right.
$$

where $\underset{\sim}{M}$ is the orthogonal and $\underset{\sim}{D}$ the diagonal matrix appearing in (2.1). Consequent7y, a necessary and sufficient condition for ordinary ellipticity, equivalent to (1.31), is given by

$$
A(\underset{\sim}{F} ; \underset{\sim}{\sim})=\frac{1}{2} \varepsilon_{i j k} \varepsilon_{p q r} \hat{m}_{i} \hat{m}_{p} H_{j q} H_{k r} \neq 0 \quad \forall \underset{\sim}{m} \in q, \underset{\sim}{H}=\underset{\sim}{H(F ; m)}, \underset{\sim}{\hat{m}}=D_{\sim}^{-1} \underset{\sim}{\sim} .
$$

Next, from (1.3) and (1.11) through (1.14), one finds that for an isotropic materia] 


$$
\begin{gathered}
c_{i j k \ell}(F)=2\left(\hat{W}_{1}+I_{1} \hat{W}_{2}\right) \delta_{i k} \delta l+4\left(\hat{W}_{2}+\hat{W}_{11}+2 I_{1} \hat{W}_{12}+I_{1}^{2} \hat{W}_{22}\right) F_{i j} F_{k \ell} \\
-2 \hat{W}_{2}\left(F_{i \ell} F_{k j}+G_{i k} \delta_{j \ell}+\delta_{i k} C_{j \ell}\right)-4\left(\hat{W}_{12}+I_{1} \hat{W}_{22}\right)\left(F_{i j} G_{k m} F_{m \ell}\right. \\
\left.+G_{i m m j} F_{k \ell} F_{k}\right)+4 \hat{W}_{22} G_{i m} F_{m j} G_{k n} F_{n \ell} .
\end{gathered}
$$

Further, (2.2), (1.28), (2.6), (1.3), (2.1), and the first of (2.3) eventually yield

$$
\left.\begin{array}{rl}
H_{i j} \underset{\sim}{(F ; m)} & =2\left[\hat{W}_{1}+\left(I_{7}-D_{k \ell} m_{k} m_{\ell}\right) \hat{W}_{2}\right] D_{i j}^{-1}+4\left(\frac{1}{2} \hat{W}_{2}+\hat{W}_{11}+2 I_{1} \hat{W}_{12}+I_{1}^{2} \hat{W}_{22}\right) m_{i} m_{j} \\
& -2 \hat{W}_{2} \delta_{i j}-4\left(\hat{W}_{12}+I_{7} \hat{W}_{22}\right)\left(m_{i} D_{j k} m_{k}+D_{i \ell} m_{\ell} m_{j}\right)+4 \hat{W}_{22} D_{i k} m_{k} D_{j \ell} m_{\ell}
\end{array}\right\}
$$

Let $\beta_{i j}, w_{i}$ and $B_{i j}, B$ be defined by

$$
\left.\begin{array}{l}
B_{i}=\hat{W}_{1}+\lambda_{i}^{2} \hat{w}_{2}, \\
w_{i}=\lambda_{i}^{2}\left(I_{1}-\lambda_{i}^{2}\right)_{i}+2 \lambda_{i}^{2}\left(I_{1}^{2}-2 I_{2}-\lambda_{i}^{4}-2 \lambda_{i}^{-2}\right)\left(\hat{w}_{11}+2 \lambda_{i}^{2} \hat{w}_{12}+\lambda_{i}^{4} \hat{w}_{22}\right) \\
\text { (no sum) },
\end{array}\right\}
$$

Note from $(2.9),(2.8),(1.14),(1.4)$ that $B_{i j}$ and $B$ are solely
functions of the $\lambda_{k}$ functions of the $\lambda_{k}$. 
Upon a lengthy computation involving (2.5), (2.7) through (2.9), (2.1), and (1.5), one verifies that for every unimodular $\underset{\sim}{F}$ and every unit vector $m$,

$$
A(\underset{\sim}{F ; M m})=\underset{\sim}{E}(\lambda ; z) \equiv 4 B_{i j} z_{i}^{2} z_{j}+B z_{1} z_{2} z_{3}, \quad z_{k} \equiv m_{k}^{2} .
$$

Suppose now that (1.10) is elliptic at $\underset{\sim}{(u, p)}$ and $\underset{\sim}{x}$. If $\lambda_{i}=\lambda_{i}(x)>0$, with $\lambda_{1} \lambda_{2} \lambda_{3}=1$, are the corresponding local principal stretches, one thus has

$$
E(\underset{\sim}{\lambda} ; z) \neq 0 \quad \forall z \in \sim,
$$

where

$$
\Lambda=\left\{\underset{\sim}{\sim} \mid z_{i} \geq 0, \quad z_{1}+z_{2}+z_{3}=1\right\} .
$$

Evidently, $\Lambda$ is the bounded and closed plane region in $z$-space whose boundary is the equilateral triangle (Figure 1) with vertices at

$$
\stackrel{\zeta}{\sim}_{1}=(1,0,0), \quad \zeta_{2}=(0,1,0), \quad \zeta_{3}=(0,0,1) .
$$

In view of $(2.11)$ and the continuity of $E(\underset{\sim}{\lambda} ; z)$ in $\underset{\sim}{z}$, this function is of one sign on $\Lambda$, so that in particular,

$$
E\left(\underset{\sim}{\left.\lambda ; \zeta_{1}\right)} E\left(\underset{\sim}{\lambda} ; \zeta_{3}\right)>0, \quad E\left(\lambda ; \zeta_{2}\right) E\left(\underset{\sim}{\lambda} ; \zeta_{3}\right)>0 .\right.
$$

Consequent1y, by virtue of (2.10), (2.9),

$$
\beta_{1} \beta_{3}>0, \beta_{2} \beta_{3}>0,
$$

and

Here and in the sequel, $\lambda$ and $z$ stand for the triplets of real numbers $\left(\lambda_{1}, \lambda_{2}, \lambda_{3}\right)$ and $\left(z_{1}, z_{2}, z_{3}\right)$, respectively. 


$$
E(\underset{\sim}{\lambda} ; z)>0 \quad \forall z \in \Lambda
$$

In order to deduce additional necessary conditions for ordinary ellipticity, we require the following

LEMMA. Let $a, b, c, d$ be real constants, with $a>0$ and $d>0$. Then

$$
a s^{3}+b s^{2} t+c s t^{2}+d t^{3}>0 \text { for } a l l \quad s \geq 0, t \geq 0, s+t=1
$$

if and only if

either $4 a c^{3}+4 d b^{3}-b^{2} c^{2}-18 a b c d+27 a^{2} d^{2}>0$ or $b>0, c>0$.

To prove this lemma, note first that (2.16) implies

$$
\theta(\xi) \equiv a \xi^{3}+b \xi^{2}+c \xi+d>0 \text { for } a 17 \quad \xi \geq 0
$$

Necessary and sufficient in order that the cubic polynomial $\theta(\xi)$ have three real zeros is that

$$
4 a c^{3}+4 d b^{3}-b^{2} c^{2}-18 a b c d+27 a^{2} d^{2} \leq 0
$$

Accordingly, (2.18) together with (2.16) imply the existence of real numbers $\xi_{i}(i=1,2,3)$ and $\bar{\xi}_{\alpha}(\alpha=1,2)$, such that

$$
\theta\left(\xi_{i}\right)=0, \quad \theta^{\prime}\left(\bar{\xi}_{\alpha}\right)=0, \quad \xi_{1} \leq \bar{\xi}_{1} \leq \xi_{2} \leq \bar{\xi}_{2} \leq \xi_{3}<0 .
$$

Thus

$$
\theta^{\prime}(\xi)=3 a \xi^{2}+2 b \xi+c=3 a\left(\xi-\bar{\xi}_{7}\right)\left(\xi-\bar{\xi}_{2}\right)
$$

${ }^{1}$ See, for example, Dickson [14], p. 47. The truth of this assertion requires merely that $a, b, c, d$ be real with $a \neq 0$. 
and therefore (2.16) and the negation of the first inequality in (2.17) necessitate

$$
\mathrm{b}=-\frac{3}{2} \mathrm{a}\left(\bar{\xi}_{1}+\bar{\xi}_{2}\right)>0, \quad \mathrm{c}=3 \mathrm{a} \bar{\xi}_{1} \bar{\xi}_{2}>0 .
$$

Hence (2.16) implies (2.17). Conversely, $b>0$ and $c>0$ at once assure that (2.16) holds. On the other hand, (2.16) also follows from the first of (2.17). Indeed, the latter mandates the existence of but one real zero of $\theta(\xi)$; this zero is negative because $a>0$ and $d>0$, so that $\theta(\xi)>0$ for all $\xi \geq 0$, which is easily seen to give (2.16). This completes the proof.

The inequality (2.15) in particular requires $E(\lambda ; z)>0$ on the side of the triangular boundary of $\Lambda$ with $z_{7}=0$, whence from (2.10),

$$
B_{22} z_{2}^{3}+B_{23} z_{2}^{2} z_{3}+B_{32} z_{2} z_{3}^{2}+B_{33} z_{3}^{3}>0 \text { for all } z_{2} \geq 0, z_{3} \geq 0, z_{2}+z_{3}=1 \text {. }
$$

The foregoing lemma thus entitles us to conclude that

$$
\left.\begin{array}{l}
\text { either } 4 B_{22} B_{32}^{3}+4 B_{33} B_{23}^{3}-B_{23}^{2} B_{32}^{2}-18 B_{22} B_{23} B_{32} B_{33}+27 B_{22}^{2} B_{33}^{2}>0 \\
\text { or } B_{23}>0, B_{32}>0 .
\end{array}\right\}
$$

Bearing in mind (2.14), we now set

$$
\rho_{i}=\beta_{j} / \lambda_{i} \beta_{1}{ }_{2} \beta_{3}>0, \omega_{i}=w_{i} / \lambda_{i} \beta_{i} \text { (no sums), }
$$

and, with the aid of (2.9), (2.21), infer the identity 


$$
\begin{aligned}
4 B_{22} B_{32}^{3} & +4 B_{33} B_{23}^{3}-B_{23}^{2} B_{32}^{2}-18 B_{22} B_{23} B_{32} B_{33}+27 B_{22}^{2} B_{33}^{2} \\
& =-\lambda_{1}^{4} \beta_{1}^{4} \beta_{2}^{2} B_{3}^{2}\left[\omega_{1}-\left(\rho_{2} \rho_{3}^{-1}+\rho_{2}^{-1} \rho_{3}\right)\right]^{2}\left(\omega_{1}^{2}-4\right) .
\end{aligned}
$$

Further, on account of (2.22), (2.9), (2.21), we draw from (2.20) that

$$
\text { either } \omega_{1}^{2}<4 \quad \text { or } \omega_{1}+\rho_{2} \rho_{3}^{-1}>0, \omega_{7}+\rho_{2}^{-1} \rho_{3}>0 \text {. }
$$

Since the second alternative in (2.23) implies

$$
\omega_{1}>-\min \left(\rho_{2} \rho_{3}^{-1}, \rho_{2}^{-1} \rho_{3}\right) \geq-1
$$

one has $\omega_{7}+2>0$. Thus and from parallel arguments applied to the edges of $\Lambda$ lying in the planes $z_{2}=0$ and $z_{3}=0$, one arrives at

$$
\omega_{i}+2>0,
$$

as necessary conditions of ordinary ellipticity.

With a view towards applying (2.15) to certain points in the interior $\AA$ of the triangular region $\Lambda$, we now suppose that $z_{i}>0$ and define functions $v_{i}$ through

$$
\left.\begin{array}{l}
v_{1}=\rho_{1}\left(\omega_{1}+\lambda_{3} \lambda_{2}^{-1} z_{2} z_{3}^{-1}+\lambda_{2} \lambda_{3}^{-1} z_{3} z_{2}^{-1}\right)+\rho_{2} \lambda_{3} \lambda_{1}^{-1} z_{1} z_{3}^{-1}+\rho_{3} \lambda_{2} \lambda_{1}^{-1} z_{1} z_{2}^{-1} \\
v_{2}=\rho_{2}\left(\omega_{2}+\lambda_{1} \lambda_{3}^{-1} z_{3} z_{1}^{-1}+\lambda_{3} \lambda_{1}^{-1} z_{1} z_{3}^{-1}\right)+\rho_{3} \lambda_{1} \lambda_{2}^{-1} z_{2} z_{1}^{-1}+\rho_{1} \lambda_{3}{ }_{2}^{-1} z_{2} z_{3}^{-1} \\
v_{3}=\rho_{3}\left(\omega_{3}+\lambda_{2} \lambda_{1}^{-1} z_{1} z_{2}^{-1}+\lambda_{1} \lambda_{2}^{-1} z_{2} z_{1}^{-1}\right)+\rho_{1} \lambda_{2} \lambda_{3}^{-1} z_{3} z_{2}^{-1}+\rho_{2} \lambda_{1} \lambda_{3}^{-1} z_{3} z_{1}^{-1}
\end{array}\right\}
$$

After some tedious algebra based on (2.10), (2.9) and involving (2.25), (2.21), one obtains 


$$
E(\lambda ; z)=-\beta_{1}^{2} \beta_{2}^{2} \beta_{3}^{2} z_{1} z_{2} z_{3}\left(v_{1}^{2}+v_{2}^{2}+v_{3}^{2}-2 v_{1} v_{2}-2 v_{2} v_{3}-2 v_{3} v_{1}\right)
$$

Consequently, (2.15) yields

$$
\text { for } z_{i}>0 \text {. }
$$

$$
v_{1}^{2}+v_{2}^{2}+v_{3}^{2}-2 v_{1} v_{2}-2 v_{2} v_{3}-2 v_{3} v_{1}<0 \text { on } \AA
$$

and thus from $(2.25),(2.24),(2.21)$ one infers that (2.15) necessitates

$$
v_{3}<v_{1}+v_{2}+2 \sqrt{v_{1} v_{2}}, \quad v_{i}>0 \text { on } \stackrel{\circ}{~} .
$$

For our present purpose it is expedient to introduce the transformation

$$
\left.\eta_{\alpha}=\eta_{\alpha} \underset{\sim}{(z ; \lambda)}\right)=\lambda_{3} \lambda_{\alpha}^{-1} z_{\alpha} z_{3}^{-1} \quad \forall \underset{\sim}{z} \in \stackrel{0}{\sim} \text { (no sum), }
$$

which is a mapping, depending parametrically on $\lambda$, of $\AA$ into the open first quadrant

$$
\Pi=\left\{\left(n_{1}, n_{2}\right) \mid n_{1}>0, n_{2}>0\right\}
$$

of the $\left(n_{1}, n_{2}\right)$-plane. Inverting (2.29) - keeping in mind that

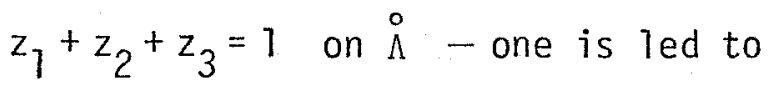

$$
\left.\begin{array}{l}
z_{\alpha}=z_{\alpha}\left(n_{1}, n_{2} ; \lambda\right)=\lambda_{\alpha} n_{\alpha}\left(\lambda_{1} n_{1}+\lambda_{2} n_{2}+\lambda_{3}\right)^{-1} \quad \text { (no sum), } \\
z_{3}=z_{3}\left(n_{1}, n_{2} ; \lambda\right)=\lambda_{3}\left(\lambda_{1} n_{1}+\lambda_{2} n_{2}+\lambda_{3}\right)^{-1} \quad \forall\left(n_{1}, n_{2}\right) \in \pi .
\end{array}\right\}
$$

Thus, (2.29) in fact constitutes a one-to-one mapping of $\AA$ onto $\pi$. Next, let functions $f$ and $g$ be defined by 


$$
\left.\begin{array}{l}
f\left(n_{1}, n_{2} ; \omega_{1}, \rho_{1}, \rho_{2}, \rho_{3}\right)=\rho_{1}\left(\omega_{1}+n_{2}+n_{2}^{-1}\right)+\rho_{2} n_{1}+\rho_{3} n_{1} n_{2}^{-1}, \\
g\left(n_{1}, n_{2} ; \omega_{1}, \omega_{2}, \rho_{1}, \rho_{2}, \rho_{3}\right)=\rho_{1} \omega_{1}+\rho_{2} \omega_{2}+2 \rho_{2} n_{1}+2 \rho_{1} n_{2} \\
\quad+2\left[f\left(n_{1}, n_{2} ; \omega_{1}, \rho_{1}, \rho_{2}, \rho_{3}\right) f\left(n_{2}, n_{1} ; \omega_{2}, \rho_{2}, \rho_{1}, \rho_{3}\right)\right]^{1 / 2} \forall\left(n_{1}, n_{2}\right) \in \mathbb{M},
\end{array}\right\}
$$

in which $\omega_{\alpha_{0}}: \rho_{j}$ are given by $(2.21),(2.8)$ and ultimately depend solely on $\lambda$. It is clear from (2.31), (2.29), (2.25), and the second of (2.28) that

$$
\begin{aligned}
& f\left(n_{1}(\underset{\sim}{z} ; \lambda), n_{2}(\underset{\sim}{z} ; \lambda) ; \omega_{1}, \rho_{1}, \rho_{2}, \rho_{3}\right)=v_{1}>0, \\
& f\left(n_{2}(\underset{\sim}{z} ; \lambda), n_{1}(\underset{\sim}{z} ; \lambda) ; w_{2}, \rho_{2}, \rho_{1}, \rho_{3}\right)=v_{2}>0 \quad \forall z \in \AA .
\end{aligned}
$$

Further, as a consequence of (2.25) and (2.30) through (2.32), the first of (2.28) is equivalent to

$$
\rho_{3} \omega_{3}<g\left(n_{1}, n_{2} ; \omega_{1}, \omega_{2}, \rho_{1}, \rho_{2}, \rho_{3}\right) \quad \forall\left(n_{1}, n_{2}\right) \in \Pi
$$

At this stage we derive from (2.33) an additional restriction on $\omega_{j}, \rho_{i}$ by choosing $n_{1}$ and $n_{2}$ so as to minimize $g\left(n_{1}, n_{2} ; \omega_{7}, \omega_{2}, \rho_{1}, \rho_{2}, \rho_{3}\right)$ on II. Thus, holding $\omega_{\alpha}, \rho_{i}$ fixed, we take $\left(n_{1}, n_{2}\right)=\left(\bar{n}_{1}, \bar{n}_{2}\right)$, where

$$
\partial g /\left.\partial n_{\alpha}\right|_{\left(\bar{n}_{1}, \bar{n}_{2}\right)}=0 .
$$

Substituting from (2.31) into (2.34), we are led to the unique solution

$\overline{1_{\text {Recall from }}(1.4) \text { that }} I_{\alpha}$, and hence $\hat{W}_{\alpha}\left(I_{7}, I_{2}\right), \hat{W}_{\alpha \beta}\left(I_{1}, I_{2}\right)$, are expressible in terms of the principal stretches. 


$$
\bar{n}_{1}=\kappa(1+\kappa)^{-1}, \quad \bar{n}_{2}=(1+\kappa)^{-1}, \quad \kappa=\left[\rho_{1}\left(\omega_{1}+2\right) / \rho_{2}\left(\omega_{2}+2\right)\right]^{1 / 2},
$$

and (2.31) now gives

$$
\left.\begin{array}{l}
f\left(\bar{n}_{1}, \bar{n}_{2} ; \omega_{1}, \rho_{1}, \rho_{2}, \rho_{3}\right)=x^{2} f\left(\bar{n}_{2}, \bar{n}_{1} ; \omega_{2}, \rho_{2}, \rho_{1}, \rho_{3}\right), \\
g\left(\bar{n}_{1}, \bar{n}_{2} ; \omega_{1}, \omega_{2}, \rho_{1}, \rho_{2}, \rho_{3}\right)=2 \rho_{3}+\left[\sqrt{\rho_{1}\left(\omega_{1}+2\right)}+\sqrt{\rho_{2}\left(\omega_{2}+2\right)}\right]^{2} .
\end{array}\right\}
$$

Finally, from (2.33), in view of the second of (2.36), follows

$$
\rho_{3}\left(\omega_{3}-2\right)<\left[\sqrt{\rho_{1}\left(\omega_{1}+2\right)}+\sqrt{\rho_{2}\left(\omega_{2}+2\right)}\right]^{2} .
$$

Proceeding similarly from the iwo cyclic permutations of (2.28), one arrives at

$$
\left.\begin{array}{l}
\rho_{1}\left(\omega_{1}-2\right)<\left[\sqrt{\rho_{2}\left(\omega_{2}+2\right)}+\sqrt{\rho_{3}\left(\omega_{3}+2\right)}\right]^{2} \\
\rho_{2}\left(\omega_{2}-2\right)<\left[\sqrt{\rho_{3}\left(\omega_{3}+2\right)}+\sqrt{\rho_{1}\left(\omega_{1}+2\right)}\right]^{2}
\end{array}\right\}
$$

We now summarize the results established in this section. To begin with, equations $(2.10),(2.15)$, and the first of (2.3) justify the assertion: for the special case of isotropy, the system of partial differential equations (1.30) is elliptic in the ordinary sense at a relaxed solution $(\underset{\sim}{u}, p)$ and at a point $\underset{\sim}{x}$ only if

Equation (2.34) merely characterizes $\left(\bar{n}_{1}, \bar{n}_{2}\right)$ as a stationary point of $g\left(n_{1}, n_{2} ; \omega_{7}, \omega_{2}, \rho_{1}, \rho_{2}, \rho_{3}\right)$; that this choice of $\left(n_{1}, n_{2}\right)$ actually minimizes $g$ on II for fixed $\lambda$-as is shown in, and essential to, the analysis in Section 3 -is irrelevant at present. Indeed, as far as our immediate objective is concerned, but for lack of motivation, $\left(\bar{n}_{1}, \bar{n}_{2}\right)$ could have been defined directly by (2.35), without recourse to $(2.34)$. 


$$
A(F ; n)>0 \quad \forall n \in \mathcal{\sim} \in \quad \underset{\sim}{\sim}=\underset{\sim}{1}+\nabla \underset{\sim}{ }(x),
$$

where $A(F ; n)$ is the determinant defined by (1.30) and involves the acoustic tensor introduced in (1.28). Moreover, in view of (2.14), $(2.24),(2.37),(2.38)$, a set of explicit necessary conditions for ordinary ellipticity in the circumstances under consideration is supplied by the inequalities

$$
\left.\begin{array}{cc}
\beta_{1} \beta_{3}>0, \quad \beta_{2} \beta_{3}>0, & \text { (a) } \\
\omega_{i}+2>0, & \text { (b) } \\
\rho_{1}\left(\omega_{1}-2\right)<\left[\sqrt{\rho_{2}\left(\omega_{2}+2\right)}+\sqrt{\rho_{3}\left(\omega_{3}+2\right)}\right]^{2} \\
\rho_{2}\left(\omega_{2}-2\right)<\left[\sqrt{\rho_{3}\left(\omega_{3}+2\right)}+\sqrt{\rho_{1}\left(\omega_{1}+2\right)}\right]^{2}, \\
\rho_{3}\left(\omega_{3}-2\right)<\left[\sqrt{\rho_{1}\left(\omega_{1}+2\right)}+\sqrt{\rho_{2}\left(\omega_{2}+2\right)}\right]^{2}
\end{array}\right\} \text { (c) }
$$

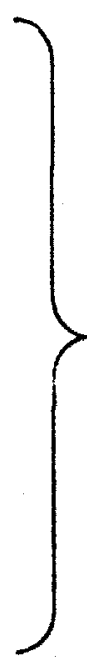

Here $\rho_{i}, \omega_{i}, \beta_{i}$ are accounted for in $(2.21),(2.8),(1.14)$ and are given by

$$
\begin{aligned}
& \rho_{i}=\beta_{i} / \lambda_{i} \beta_{1} \beta_{2} \beta_{3}, \quad \omega_{i}=w_{i} / \lambda_{i} \beta_{i} \quad \text { (no sums), } \\
& \beta_{i}=\hat{W}_{1}\left(I_{1}, I_{2}\right)+\lambda_{i}^{2} \hat{W}_{2}\left(I_{1}, I_{2}\right), \\
& W_{i}=\lambda_{i}^{2}\left(I_{1}-\lambda_{i}^{2}\right) \beta_{i}+2 \lambda_{i}^{2}\left(I_{1}^{2}-2 I_{2}-\lambda_{i}^{4}-2 \lambda_{i}^{-2}\right)\left[\hat{W}_{11}\left(I_{1}, I_{2}\right)\right. \\
& \left.+2 \lambda_{i}^{2} \hat{W}_{12}\left(I_{1}, I_{2}\right)+\lambda_{i}^{4} \hat{W}_{22}\left(I_{1}, I_{2}\right)\right] \quad \text { (no sum), }
\end{aligned}
$$

where $\lambda_{i}=\lambda_{i}(\underset{\sim}{x})$ are the local principal stretches associated with the displacement field $\underset{\sim}{u}$, while $\hat{W}_{\alpha}\left(I_{1}, I_{2}\right)$ and $\hat{W}_{\alpha \beta}\left(I_{1}, I_{2}\right)$ are the 
corresponding local values at $I_{\alpha}=I_{\alpha}(\underset{\sim}{x})$ of the first and second partial derivatives of the strain-energy density with respect to the deformation invariants.

With a view towards expressing $\beta_{i}, w_{i}$ in terms of the partial derivatives of the strain-energy density with respect to the principal stretches, we note from $(1.4),(1.5)$ that

$$
\begin{aligned}
& I_{1}=\stackrel{\star}{I}_{1}\left(\lambda_{1}, \lambda_{2}\right)=\lambda_{1}^{2}+\lambda_{2}^{2}+\lambda_{1}^{-2} \lambda_{2}^{-2}, \\
& I_{2}=\stackrel{*}{I}_{2}\left(\lambda_{1}, \lambda_{2}\right)=\lambda_{1}^{2} \lambda_{2}^{2}+\lambda_{1}^{-2}+\lambda_{2}^{-2} .
\end{aligned}
$$

We now define

$$
\stackrel{*}{W}\left(\lambda_{1}, \lambda_{2}\right)=\hat{W}\left(\stackrel{*}{I}_{1}\left(\lambda_{1}, \lambda_{2}\right), \stackrel{*}{I}_{2}\left(\lambda_{1}, \lambda_{2}\right)\right),
$$

and adopt the notation

$$
\stackrel{*}{W}_{\alpha}\left(\lambda_{1}, \lambda_{2}\right)=\frac{\partial \stackrel{*}{W}\left(\lambda_{1}, \lambda_{2}\right)}{\partial \lambda_{\alpha}}, \quad \stackrel{*}{W}_{\alpha \beta}\left(\lambda_{1}, \lambda_{2}\right)=\frac{\partial^{2}{ }^{*}\left(\lambda_{1}, \lambda_{2}\right)}{\partial \lambda_{\alpha} \partial \lambda_{\beta}} .
$$

From $(1.5),(1.14)$ and the chain-rule then follow

$$
\begin{aligned}
& \stackrel{*}{W}_{\alpha}\left(\lambda_{1}, \lambda_{2}\right)=2\left(\lambda_{\alpha}-\lambda_{3}^{2} \lambda_{\alpha}^{-1}\right)\left(\hat{W}_{1}+\lambda_{3}^{-2} \lambda_{\alpha}^{-2} \hat{W}_{2}\right) \text { (no sum), } \\
& \stackrel{\star}{\hat{W}_{\alpha \alpha}}\left(\lambda_{1}, \lambda_{2}\right)=2\left(1+3 \lambda_{3}^{2} \lambda_{\alpha}^{-2}\right)\left(\hat{W}_{1}+\lambda_{3}^{-2} \lambda_{\alpha}^{-2} \hat{W}_{2}\right) \\
& +4\left(\lambda_{\alpha}-\lambda_{3}^{2} \lambda_{\alpha}^{-1}\right)^{2}\left(\hat{W}_{11}+2 \lambda_{3}^{-2} \lambda_{\alpha}^{-2} \hat{W}_{12}+\lambda_{3}^{-4} \lambda_{\alpha}^{-4} \hat{W}_{22}\right) \text { (no sum), } \\
& \stackrel{\star \star ⿱ ⺌}{W}_{12}\left(\lambda_{1}, \lambda_{2}\right)=4 \lambda_{3}^{3}\left(\hat{W}_{1}+\lambda_{3}^{-4} \hat{W}_{2}\right)+4 \lambda_{3}\left(\lambda_{1}^{2}-\lambda_{3}^{2}\right)\left(\lambda_{2}^{2}-\lambda_{3}^{2}\right)\left[\hat{W}_{11}+\left(\lambda_{1}^{2}+\lambda_{2}^{2}\right) \hat{W}_{12}\right. \\
& \left.+\lambda_{3}^{-2} \hat{W}_{22}\right]
\end{aligned}
$$

in which 


$$
\begin{array}{r}
\lambda_{3}=\left(\lambda_{1} \lambda_{2}\right)^{-1}, \hat{W}_{\alpha}=\hat{W}_{\alpha}\left(\stackrel{I}{I}_{1}\left(\lambda_{1}, \lambda_{2}\right), \stackrel{\star}{I}_{2}\left(\lambda_{1}, \lambda_{2}\right)\right), \\
\hat{W}_{\alpha \beta}=\hat{W}_{\alpha \beta}\left(\hat{I}_{1}^{*}\left(\lambda_{1}, \lambda_{2}\right), \hat{I}_{2}\left(\lambda_{1}, \lambda_{2}\right)\right) .
\end{array}
$$

By means of $(2.42),(2.43),(2.46)$, and $(2.47)$, we can verify that

$$
\begin{aligned}
& \beta_{1}=\stackrel{*}{\beta}_{1}\left(\lambda_{1}, \lambda_{2}\right)=\frac{1}{2}\left(\lambda_{2}^{2}-\lambda_{3}^{2}\right)^{-1} \lambda_{2} \stackrel{\star}{W}_{2}\left(\lambda_{1}, \lambda_{2}\right), \\
& w_{1}=\stackrel{*}{w_{1}}\left(\lambda_{1}, \lambda_{2}\right)=-\left(\lambda_{2}^{2}-\lambda_{3}^{2}\right)^{-1} \lambda_{2}^{-1} \stackrel{\star}{W}_{2}\left(\lambda_{1}, \lambda_{2}\right)+\frac{1}{2} \lambda_{3}^{-2} w_{22}^{*}\left(\lambda_{1}, \lambda_{2}\right), \lambda_{3}=\left(\lambda_{1} \lambda_{2}\right)^{-1} \\
& \left(\lambda_{2} \neq \lambda_{3}\right) \text {, } \\
& \beta_{2}=\stackrel{*}{\beta}_{2}\left(\lambda_{1}, \lambda_{2}\right)=\frac{1}{2}\left(\lambda_{1}^{2}-\lambda_{3}^{2}\right)^{-1} \lambda_{1} \stackrel{*}{W}_{1}\left(\lambda_{1}, \lambda_{2}\right), \\
& w_{2}=\stackrel{*}{w_{2}}\left(\lambda_{1}, \lambda_{2}\right)=-\left(\lambda_{1}^{2}-\lambda_{3}^{2}\right)^{-1} \lambda_{1}^{-1} \stackrel{*}{w}_{1}\left(\lambda_{1}, \lambda_{2}\right)+\frac{1}{2} \lambda_{3}^{-2} \stackrel{*}{w}_{11}\left(\lambda_{1}, \lambda_{2}\right), \lambda_{3}=\left(\lambda_{1} \lambda_{2}\right)^{-1} \\
& \left(\lambda_{3} \neq \lambda_{1}\right) \text {, } \\
& \beta_{3}=\stackrel{*}{\beta}_{3}\left(\lambda_{1}, \lambda_{2}\right)=\frac{1}{2}\left(\lambda_{1}^{2}-\lambda_{2}^{2}\right)^{-1}\left[\lambda_{1} \stackrel{\star *}{W}_{1}\left(\lambda_{1}, \lambda_{2}\right)-\lambda_{2} \stackrel{\star *}{W}_{2}\left(\lambda_{1}, \lambda_{2}\right)\right], \\
& w_{3}=\stackrel{*}{w}_{3}\left(\lambda_{1}, \lambda_{2}\right)=\left(\lambda_{1}^{2}-\lambda_{2}^{2}\right)^{-1}\left[\lambda_{2}^{-1} \stackrel{*}{W}_{2}\left(\lambda_{1}, \lambda_{2}\right)-\lambda_{1}^{-1} \stackrel{W}{1}_{1}\left(\lambda_{1}, \lambda_{2}\right)\right] \\
& +\frac{1}{2}\left[\lambda_{2}^{-2} \stackrel{*}{W}_{11}\left(\lambda_{1}, \lambda_{2}\right)+\lambda_{1}^{-2} \stackrel{*}{W}_{22}\left(\lambda_{1}, \lambda_{2}\right)-2 \lambda_{1}^{-1} \lambda_{2}^{-1} \stackrel{*}{W}_{12}\left(\lambda_{1}, \lambda_{2}\right)\right]\left(\lambda_{1} \neq \lambda_{2}\right) .
\end{aligned}
$$

Equations (2.48) exclude the case of coalescent principal stretches. In order to derive representations for $\beta_{i}, w_{i}$ applicable when two of the $\lambda_{i}$ are equal, one makes use of $(2.44),(2.43)$ to infer

$$
\stackrel{*}{W}\left(\lambda_{1}, \lambda_{2}\right)=\stackrel{*}{W}\left(\lambda_{2}, \lambda_{1}\right)=\stackrel{*}{W}\left(\lambda_{2}, \lambda_{3}\right)=\stackrel{*}{W}\left(\lambda_{3}, \lambda_{1}\right), \lambda_{3}=\left(\lambda_{1} \lambda_{2}\right)^{-1},
$$

whence 
$\stackrel{\star}{W}_{1}\left(\lambda_{1}, \lambda_{2}\right)=\stackrel{\star}{W}_{2}\left(\lambda_{2}, \lambda_{1}\right), \stackrel{\star}{W}_{11}\left(\lambda_{1}, \lambda_{2}\right)=\stackrel{\star}{W}_{22}\left(\lambda_{2}, \lambda_{1}\right), \stackrel{\star}{W}_{12}\left(\lambda_{1}, \lambda_{2}\right)=\stackrel{\star}{W}_{12}\left(\lambda_{2}, \lambda_{1}\right),(a)$

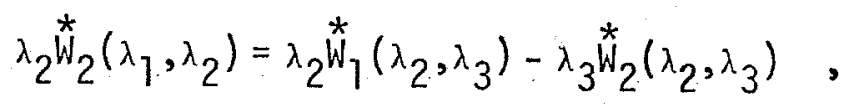

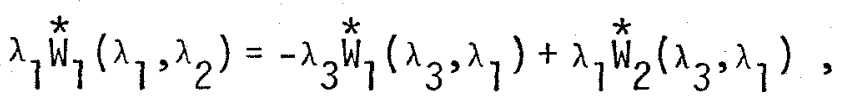

$\lambda_{2}^{2} \mathbb{W}_{22}^{*}\left(\lambda_{1}, \lambda_{2}\right)=$

$2 \lambda_{3}{ }_{2}^{*}\left(\lambda_{2}, \lambda_{3}\right)+\lambda_{2}^{2} \underset{W_{11}}{*}\left(\lambda_{2}, \lambda_{3}\right)+\lambda_{3}^{2} \underset{W_{22}}{\stackrel{*}{W}}\left(\lambda_{2}, \lambda_{3}\right)-2 \lambda_{1}^{-1} \mathbb{W}_{12}^{*}\left(\lambda_{2}, \lambda_{3}\right)$,

$\lambda_{1}^{2} \stackrel{\star}{W}_{17}\left(\lambda_{1}, \lambda_{2}\right)=$

$2 \lambda_{3} \stackrel{\star}{W}_{1}\left(\lambda_{3}, \lambda_{7}\right)+\lambda_{3}^{2} \stackrel{*}{W_{11}}\left(\lambda_{3}, \lambda_{1}\right)+\lambda_{1}^{2} \stackrel{\star}{W}_{22}\left(\lambda_{3}, \lambda_{1}\right)-2 \lambda_{2}^{-1} \mathbb{W}_{12}^{*}\left(\lambda_{3}, \lambda_{1}\right)$,

$$
\lambda_{3}=\left(\lambda_{1} \lambda_{2}\right)^{-1} \text {. }
$$

(2.49)

Substitution from (b) of (2.49) into (2.48) gives

$$
\left.\begin{array}{ll}
{ }_{\beta_{1}}^{*}\left(\lambda_{1}, \lambda_{2}\right)={\stackrel{*}{\beta_{3}}}_{3}\left(\lambda_{2}, \lambda_{3}\right), & \stackrel{*}{w_{1}}\left(\lambda_{1}, \lambda_{2}\right)=\stackrel{*}{w_{3}}\left(\lambda_{2}, \lambda_{3}\right), \\
{ }_{\beta_{2}}^{*}\left(\lambda_{1}, \lambda_{2}\right)={ }_{\beta_{3}}^{*}\left(\lambda_{3}, \lambda_{1}\right), & \stackrel{*}{w_{2}}\left(\lambda_{1}, \lambda_{2}\right)=\stackrel{*}{w_{3}}\left(\lambda_{3}, \lambda_{1}\right), \lambda_{3}=\left(\lambda_{1} \lambda_{2}\right)^{-1}
\end{array}\right\}
$$

Further, on account of the first two of (a) in (2.49), an obvious limit

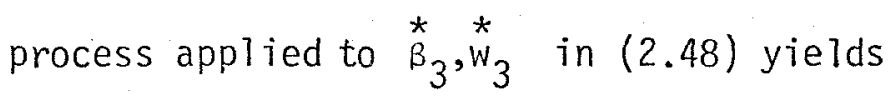

$$
{ }_{\beta_{3}}^{*}(\lambda, \lambda)=\frac{1}{2} \lambda^{2 *} W_{3}(\lambda, \lambda)=\frac{1}{4}\left[\lambda \lambda^{-1} \mathbb{W}_{1}^{*}(\lambda, \lambda)+\stackrel{*}{W}_{11}^{*}(\lambda, \lambda)-{ }_{W}^{*} W_{12}(\lambda, \lambda)\right] \quad(\lambda>0) .
$$

Consequently, if one sets

$$
\beta\left(\lambda_{1}, \lambda_{2}\right)=\left\{\begin{array}{ll}
\frac{1}{4}\left[\lambda^{-1} \mathbb{W}_{1}(\lambda, \lambda)+\mathbb{W}_{11}^{*}(\lambda, \lambda)-\stackrel{*}{W}_{12}(\lambda, \lambda)\right] & \text { for } \lambda_{1}=\lambda_{2}=\lambda, \\
\frac{1}{2}\left(\lambda_{1}^{2}-\lambda_{2}^{2}\right)^{-1}\left[\lambda_{1} \stackrel{*}{W}_{1}\left(\lambda_{1}, \lambda_{2}\right)-\lambda_{2} \mathbb{W}_{2}\left(\lambda_{1}, \lambda_{2}\right)\right] \text { for } \lambda_{1} \neq \lambda_{2},
\end{array}\right\}
$$




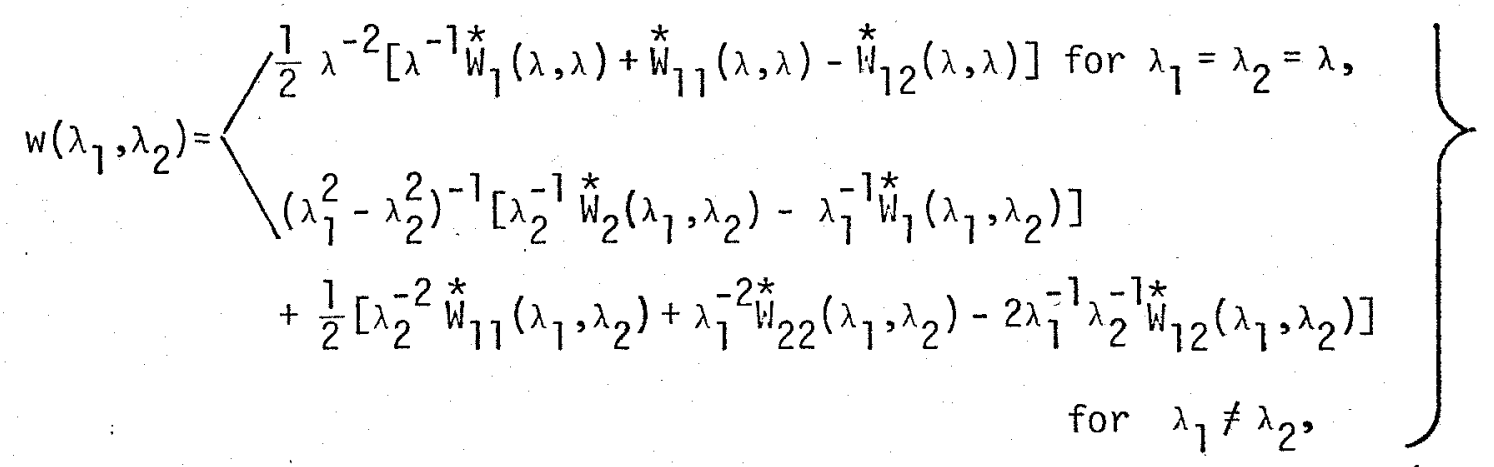

then $(2.48),(2.50),(2.51)$ justify

$$
\left.\begin{array}{l}
\beta_{1}=\beta\left(\lambda_{2}, \lambda_{3}\right), \quad \beta_{2}=\beta\left(\lambda_{3}, \lambda_{1}\right), \quad \beta_{3}=\beta\left(\lambda_{1}, \lambda_{2}\right), \\
w_{1}=w\left(\lambda_{2}, \lambda_{3}\right), \quad w_{2}=w\left(\lambda_{3}, \lambda_{1}\right), \quad w_{3}=w\left(\lambda_{1}, \lambda_{2}\right),
\end{array}\right\}
$$

which accommodate all $\lambda_{i}>0$. Finally, from the second and fourth functional relations in (b) of (2.49) and the second in (a), one finds that $\beta, W$ may more convenientiy be written as

$$
\left.\begin{array}{l}
\beta\left(\lambda_{1}, \lambda_{2}\right)=\left\{\begin{array}{ll}
\frac{1}{8} \stackrel{*}{W}_{11}\left(\lambda, \lambda^{-2}\right) & \text { for } \lambda_{1}=\lambda_{2}=\lambda, \\
-\frac{1}{2} \lambda_{2}\left(\lambda_{1}^{2}-\lambda_{2}^{2}\right)^{-1} \stackrel{*}{W}_{1}^{*}\left(\lambda_{2}, \lambda_{3}\right) & \text { for } \lambda_{1} \neq \lambda_{2}, \lambda_{3}=\left(\lambda_{1} \lambda_{2}\right)^{-1}
\end{array}\right\} \\
W\left(\lambda_{1}, \lambda_{2}\right)=\left\{\begin{array}{ll}
\frac{1}{4} \lambda^{-2} W_{11}^{*}\left(\lambda, \lambda^{-2}\right) & \text { for } \lambda_{1}=\lambda_{2}=\lambda, \\
\lambda_{2}^{-1}\left(\lambda_{1}^{2}-\lambda_{2}^{2}\right)^{-1} \stackrel{*}{W}_{1}^{*}\left(\lambda_{2}, \lambda_{3}\right)+\frac{1}{2} \lambda_{1}^{-2} W_{11}^{*}\left(\lambda_{2}, \lambda_{3}\right) & \text { for } \lambda_{1} \neq \lambda_{2}, \lambda_{3}=\left(\lambda_{1} \lambda_{2}\right)^{-1}
\end{array}\right\}
\end{array}\right\}
$$

Equations (2.53), (2.54) comprise the desired intrinsic representations of $\beta_{j}$ and $w_{i}$ in terms of the $\lambda_{j}$ and the requisite partial derivatives of $\underset{W}{*}$. Moreover, $\beta_{i}$ and $w_{i}$ now exhibit the cyclic 
symmetry inherent in (2.53). Each of (a), (b), and (c) in the ellipticity conditions (2.40) is carried into itself by a cyclic permutation of the indices. ${ }^{1}$. For the purpose of writing (2.40) as a set of inequalities symmetric with respect to $\left(\lambda_{1}, \lambda_{2}, \lambda_{3}\right)$, we define functions $\rho, \omega, \varphi_{i}$ through

$$
\left.\begin{array}{c}
\rho\left(\lambda_{1}, \lambda_{2}\right)=\lambda_{1} \lambda_{2} / \beta\left(\lambda_{2}, \lambda_{3}\right) \beta\left(\lambda_{3}, \lambda_{1}\right), \\
\omega\left(\lambda_{1}, \lambda_{2}\right)=\lambda_{1} \lambda_{2} w\left(\lambda_{1}, \lambda_{2}\right) / \beta\left(\lambda_{1}, \lambda_{2}\right), \quad \lambda_{3}=\left(\lambda_{1} \lambda_{2}\right)^{-1},
\end{array}\right\}
$$

with $\beta, W$ given by (2.54). Because of $(2.55),(2.53),(2.41)$, we have

$$
\begin{aligned}
& \rho\left(\lambda_{1}, \lambda_{2}\right)=\rho_{3}, \rho\left(\lambda_{2}, \lambda_{3}\right)=\rho_{1}, \rho\left(\lambda_{3}, \lambda_{1}\right)=\rho_{2} \\
& \omega\left(\lambda_{1}, \lambda_{2}\right)=\omega_{3}, \quad \omega\left(\lambda_{2}, \lambda_{3}\right)=\omega_{1}, \omega\left(\lambda_{3}, \lambda_{1}\right)=\omega_{2},
\end{aligned}
$$

and thus, on appealing to $(2.53),(2.56)$, conclude that

$$
\begin{aligned}
& \varphi_{1}\left(\lambda_{1}, \lambda_{2}\right)=\beta_{1} \beta_{2}, \quad \varphi_{1}\left(\lambda_{2}, \lambda_{3}\right)=\beta_{2} \beta_{3}, \quad \varphi_{1}\left(\lambda_{3}, \lambda_{1}\right)=\beta_{3} \beta_{1}, \\
& \varphi_{2}\left(\lambda_{1}, \lambda_{2}\right)=\omega_{3}+2, \quad \varphi_{2}\left(\lambda_{2}, \lambda_{3}\right)=\omega_{1}+2, \quad \varphi_{2}\left(\lambda_{3}, \lambda_{1}\right)=\omega_{2}+2, \\
& \varphi_{3}\left(\lambda_{1}, \lambda_{2}\right)=\rho_{3}\left(2-\omega_{3}\right)+\left[\sqrt{\rho_{1}}\left(\omega_{1}+2\right)+\sqrt{\rho_{2}\left(\omega_{2}+2\right)}\right]^{2},
\end{aligned}
$$

After one cyclic step, (a) of (2.40) becomes $\beta_{2} \beta_{1}>0, \beta_{3} \beta_{7}>0$, which is equivalent to (a). 


$$
\begin{aligned}
& \varphi_{\dot{3}}\left(\lambda_{2}, \lambda_{3}\right)=\rho_{1}\left(2-\omega_{1}\right)+\left[\sqrt{\rho_{2}\left(\omega_{2}+2\right)}+\sqrt{\rho_{3}\left(\omega_{3}+2\right)}\right]^{2}, \\
& \varphi_{3}\left(\lambda_{3}, \lambda_{1}\right)=\rho_{2}\left(2-\omega_{2}\right)+\left[\sqrt{\rho_{3}\left(\omega_{3}+2\right)}+\sqrt{\rho_{1}\left(\omega_{1}+2\right)}\right]^{2} .
\end{aligned}
$$

Hence, the necessary ellipticity conditions (2.40) are equivalent to the nine symmetric inequalities

$$
\varphi_{i}\left(\lambda_{1}, \lambda_{2}\right)>0, \varphi_{i}\left(\lambda_{2}, \lambda_{3}\right)>0, \varphi_{j}\left(\lambda_{3}, \lambda_{1}\right)>0,
$$

with $\varphi_{i}$ supplied by (2.54) through (2.56).

We observe that (2.58) remains invariant under cyclic permutations of $\left(\lambda_{1}, \lambda_{2}, \lambda_{3}\right)$. In addition, we gather from (2.52), (a) of (2.49), and (2.55) that

$$
\begin{aligned}
& \beta\left(\lambda_{1}, \lambda_{2}\right)=\beta\left(\lambda_{2}, \lambda_{1}\right), \omega\left(\lambda_{1}, \lambda_{2}\right)=w\left(\lambda_{2}, \lambda_{1}\right), \\
& \rho\left(\lambda_{1}, \lambda_{2}\right)=\rho\left(\lambda_{2}, \lambda_{1}\right), \omega\left(\lambda_{1}, \lambda_{2}\right)=\omega\left(\lambda_{2}, \lambda_{1}\right) .
\end{aligned}
$$

Further, (2.56) now gives

$$
\varphi_{i}\left(\lambda_{1}, \lambda_{2}\right)=\varphi_{j}\left(\lambda_{2}, \lambda_{1}\right)
$$

so that the ellipticity conditions (2.58) are in fact invariant under a]1 permutations of the principal stretches and thus reflect the isotropy of the material.

${ }^{1}$ Clearly, (a) of $(2.40)$ implies $\beta_{1} \beta_{2}>0$, whence $(2.40)$ is equivalent to the set of nine conditions consisting of (2.40) augmented by $\beta_{1} \beta_{2}>0$. 
3. Sufficiency of the conditions necessary for ordinary ellipticity.

Explicit necessary and sufficient conditions for strong ellipticity in the case of isotropy.

In this section we show first that the set of necessary ellipticity conditions (2.40) is also sufficient in order that the system of partial differential equations (1.10) be elliptic in the ordinary sense, provided the solid at hand is isotropic. Thereafter, we prove that (2.40) with (a) replaced by $\beta_{i}>0$ are necessary and sufficient conditions for for the strong ellipticity of (1.10). Finally, at the end of the section, we note circumstances in which the conditions of ordinary and strong el7ipticity coalesce.

Aiming at the first of the foregoing three objectives, we now suppose $(2.40)$ holds and demonstrate that then

$$
E(\lambda ; z)>0 \quad \forall z \in \Lambda
$$

where $E(\underset{\sim}{\lambda} ; z)$ and $\Lambda$ are given by $(2.10),(2.9),(2.8),(2.12)$. It is evident from (2.5) and (2.10) that (3.1), in turn, will suffice to assure the local ordinary ellipticity of $(1.10)$ at the relaxed solution under consideration.

From (a) of (2.40), in conjunction with (2.9) and (2.41), directly follows

$$
B_{i i}>0 \quad(\text { no sum }), \rho_{i}>0
$$

Further, since $\omega_{1}+2>0$ according to (b) of (2.40),

$$
\text { either }-2<\omega_{1}<2 \text { or } \quad \omega_{1} \geq 2 \text {. }
$$


On the other hand, (3.3) and the second of (3.2) permit the inference either $-2<\omega_{1}<2 \leq \rho_{2} \rho_{3}^{-1}+\rho_{2}^{-1} \rho_{3} \quad$ or $\quad \omega_{1}+\rho_{2} \rho_{3}^{-1}>2, \omega_{1}+\rho_{2}^{-1} \rho_{3}>2$, so that, because of (a) in (2.40),

$$
\left.\begin{array}{l}
\text { either } \quad-\lambda_{1}^{4} \beta_{1}^{4} \beta_{2}^{2} \beta_{3}^{2}\left[\omega_{1}-\left(\rho_{2} \rho_{3}^{-1}+\rho_{2}^{-1} \rho_{3}\right)\right]^{2}\left(\omega_{1}^{2}-4\right)>0 \\
\quad \text { or } \quad \lambda_{1} \beta_{1} \beta_{3}\left(\omega_{1}+\rho_{2} \rho_{3}^{-1}\right)>0, \quad \lambda_{1} \beta_{1} \beta_{2}\left(\omega_{1}+\rho_{2}^{-1} \rho_{3}\right)>0 .
\end{array}\right\}
$$

Next, recalling (2.22), (2.41), and (2.9), we see that (3.4) is equivalerit to (2.20). But (2.20), as a consequence of the first of (3.2) and the lemma established in Section 2, implies (2.19), which then - by virtue of $(2.10)$ - gives

$$
E(\lambda ; z)>0 \text { for every } \underset{\sim}{z} \in \Lambda \text { with } z_{1}=0 \text {. }
$$

Proceeding similarly from the remaining two inequalities in (b) of (2.40), one thus obtains

$$
E(\lambda ; z)>0 \quad \forall \underset{\sim}{\sim} \in \partial \Lambda \equiv \Lambda-\Lambda,
$$

whence (3.1) is true on the edges of $\Lambda$.

We have yet to confirm the inequality (3.1) on the interior $\AA$. As a first step in this direction, we show presently that

$$
g\left(n_{1}, n_{2} ; \omega_{7}, \omega_{2}, \rho_{1}, \rho_{2}, \rho_{3}\right) \geq g\left(\bar{n}_{1}, \bar{n}_{2} ; \omega_{1}, w_{2}, \rho_{1}, \rho_{2}, \rho_{3}\right) \quad \forall\left(n_{1}, n_{2}\right) \in \mathbb{I},
$$

where $g$ is the function introduced in (2.31), while $\bar{n}_{\alpha}$ is given by (2.35); as before, II denotes the open first quadrant of the $\left(n_{7}, n_{2}\right)$ plane. Accordingly, we are to prove that the choice $\left(n_{7}, n_{2}\right)=\left(\bar{n}_{7}, \bar{n}_{2}\right)$, 
which is already known from (2.34) to render g stationary for fixed $\lambda$, in fact minimizes this function on $\pi$.

In order to verify the above claim, we note that the first of (2.3T) allows us to write

$$
\left.\begin{array}{l}
f\left(n_{1}, n_{2} ; \omega_{1}, \rho_{1}, \rho_{2}, \rho_{3}\right)=\psi_{1}^{2}+\rho_{1} n_{2}^{-1}\left(1-n_{2}\right)^{2}+\rho_{2} n_{1}+\rho_{3} n_{1} n_{2}^{-1}, \\
f\left(n_{2}, n_{1} ; \omega_{2}, \rho_{2}, \rho_{1}, \rho_{3}\right)=\psi_{2}^{2}+\rho_{2} n_{1}^{-1}\left(1-n_{1}\right)^{2}+\rho_{1} n_{2}+\rho_{3} n_{1}^{-1} n_{2},
\end{array}\right\}
$$

provided

$$
\psi_{\alpha}=\sqrt{\rho_{\alpha}\left(\omega_{\alpha}+2\right)} \text { (no sum). }
$$

A lengthy, but straightforward, computation then confirms that

$$
\begin{aligned}
& f\left(n_{1}, n_{2} ; \omega_{1}, \rho_{1}, \rho_{2}, \rho_{3}\right) f\left(n_{2}, n_{1} ; \omega_{2}, \rho_{2}, \rho_{1}, \rho_{3}\right) \\
& =\left[\rho_{3}+\psi_{1} \psi_{2}+\rho_{1}\left(1-n_{2}\right)+\rho_{2}\left(1-n_{1}\right)\right]^{2} \\
& +\left(\rho_{3} \rho_{1} n_{1}^{-1}+\rho_{2} \rho_{3} n_{2}^{-1}+\rho_{1} \rho_{2} \eta_{1}^{-1} n_{2}^{-1}\right)\left(n_{1}+n_{2}-1\right)^{2} \\
& +\rho_{3} \eta_{1}^{-1} n_{2}^{-1}\left(n_{2} \psi_{1}-n_{1} \psi_{2}\right)^{2}+\rho_{1} n_{2}\left[\psi_{1}-n_{2}^{-1}\left(1-n_{2}\right) \psi_{2}\right]^{2} \\
& +\rho_{2} \eta_{1}\left[\psi_{2}-n_{1}^{-1}\left(1-n_{1}\right) \psi_{1}\right]^{2} .
\end{aligned}
$$

Since $\rho_{i}>0$ and $\psi_{\alpha}>0$ by $(a),(b)$ of $(2.40)$, all terms in the righthand member of (3.9) are non-negative, and

$$
\begin{aligned}
& f\left(n_{1}, n_{2} ; \omega_{1}, \rho_{1}, \rho_{2}, \rho_{3}\right) f\left(n_{2}, n_{7} ; \omega_{2}, \rho_{2}, \rho_{1}, \rho_{3}\right) \\
& \geq\left[\rho_{3}+\psi_{1} \psi_{2}+\rho_{1}\left(1-n_{2}\right)+\rho_{2}\left(1-n_{1}\right)\right]^{2} \quad \forall\left(n_{1}, n_{2}\right) \in \Pi .
\end{aligned}
$$

Combining this lower bound with (3.8) and the second of (2.31), we are 
led to

$$
\begin{aligned}
g\left(n_{1}, n_{2} ; \omega_{1}, \omega_{2}, \rho_{1}, \rho_{2}, \rho_{3}\right) \geq 2 \rho_{3}+\left[\sqrt{\rho_{1}\left(\omega_{1}+2\right)}\right. & \left.+\sqrt{\rho_{2}\left(\omega_{2}+2\right)}\right]^{2} \\
& \forall\left(n_{1}, n_{2}\right) \in \pi .
\end{aligned}
$$

Finally, from the second of (2.36), it is evident that (3.10) is equivalent to (3.6).

Next, from (3.10) and the last inequality in (c) of (2.40) at once follows (2.33). Furthermore, (a) and (b) of (2.40) assure that $v_{i}>0$ on $\Lambda$, if the $v_{i}$ are the three functions defined in (2.25). But (2.33), with the aid of $(2.31),(2.29),(2.32)$, and $(2.25)$, is then readily found to imply (2.28). Parallel considerations applied to the first two inequalities in (c) of $(2.40)$ result in

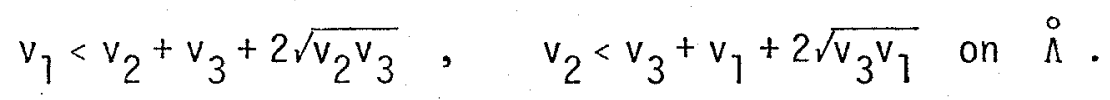

Therefore, as $v_{i}$ is positive,

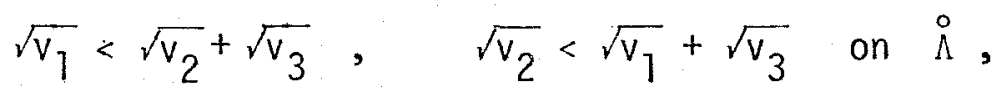

and thus

$$
\left(\sqrt{v_{1}}-\sqrt{v_{2}}\right)^{2}<v_{3} \text { on } \AA
$$

The inequalities (2.28) and (3.12), in turn, enable us to conclude that

$$
-2 \sqrt{v_{1} v_{2}}<v_{3}-v_{1}-v_{2}<2 \sqrt{v_{1} v_{2}} \text { on } \AA \text {, }
$$

whence we arrive at (2.27). The latter, because of (2.26) and (a) in (2.40), yields

$$
E(\underset{\sim}{\lambda} ; z)>0 \quad \forall \underset{\sim}{z} \in \AA
$$


which - together with (3.5) - justifies our original claim (3.1).

We are now in a position to assert that the set of inequalities (2.40), or alternatively its symmetric equivalent (2.58), are both necessary and sufficient for the ordinary ellipticity of the system of partial differential equations (1.10) at a relaxed solution $(u, p)$ and a point $x$, provided $\lambda_{i}=\lambda_{i}(\underset{\sim}{x})$ are the local principal stretches. Our next task is to derive from (1.49) an explicit set of conditions necessary and sufficient for the strong ellipticity of (1.10) in the special case of isotropy. To this end we recall (2.2), (2.3) and note that

$$
\hat{n}=\underset{\sim}{N} \underset{\sim}{T}, \quad \underset{\sim}{\sim}={\underset{\sim}{N}}^{-1} \underset{\sim}{H} N^{-T} ;
$$

hence

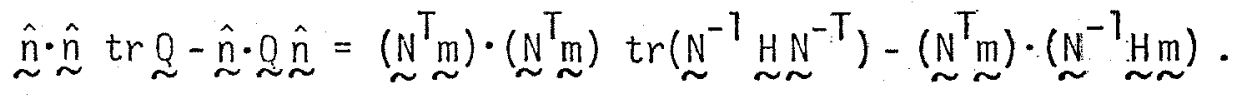

Further, from (2.2), (2.1), and (1.3) follow

$$
\underset{\sim}{N} \mathbb{N}^{\top}=\stackrel{D}{\sim}^{-1}, \operatorname{tr}\left({\underset{\sim}{N}}^{-1} \underset{\sim}{\sim} \mathbb{\sim}^{-\top}\right)=\operatorname{tr}(\underset{\sim}{D} \underset{\sim}{H}),
$$

so that (3.14) gives

$$
\hat{n} \cdot \underset{\sim}{\hat{n}} \operatorname{tr} \underset{\sim}{Q}-\underset{\sim}{\hat{n}} \cdot \underset{\sim}{\hat{n}}=\underset{\sim}{m} \cdot\left(D_{\sim}^{-1} m\right) \operatorname{tr}(\underset{\sim}{D})-\underset{\sim}{m} \cdot \underset{\sim}{H} \mathbb{\sim}
$$

Let us define a matrix $\left[L_{i j}\right]$ in terms of the $\beta_{i}, W_{i}$ in (2.8) by setting

$$
\begin{aligned}
& L_{i j}=\lambda_{i}^{-2}\left(\beta_{1}+\beta_{2}+\beta_{3}-\beta_{i}\right) \quad \text { (no sum), } \\
& L_{12}=L_{21}=\frac{1}{2}\left(\lambda_{1}^{-2} \beta_{1}+\lambda_{2}^{-2} \beta_{2}+w_{3}\right), \\
& L_{23}=L_{32}=\frac{1}{2}\left(\lambda_{2}^{-2} \beta_{2}+\lambda_{3}^{-2} \beta_{3}+w_{1}\right), \\
& L_{31}=L_{13}=\frac{1}{2}\left(\lambda_{3}^{-2} \beta_{3}+\lambda_{1}^{-2} \beta_{1}+w_{2}\right) .
\end{aligned}
$$


A considerable amount of algebra, based on (2.7), (2.1), (2.8), and involving $(2.3),(1.5),(3.16)$, enables us to deduce

$$
m \cdot\left(D_{\sim}^{-1} \underset{\sim}{m}\right) \operatorname{tr}(\underset{\sim}{D} H)-\underset{\sim}{m} \cdot \underset{\sim}{H} \underset{\sim}{m}=2 K(\lambda ; z)
$$

provided

$$
K \underset{\sim}{\lambda ; z)}=L_{i j} z_{i} z_{j}, \quad z_{k}=m_{k}^{2}
$$

Consequent7y, applying (1.31), (2.10) to the first inequality in (1.49), and $(3.15),(3.17)$ to the second, we obtain

$$
E(\underset{\sim}{\lambda ; z})>0, \quad K(\underset{\sim}{\lambda} ; z)>0 \quad \forall \underset{\sim}{z} \in \Lambda \quad
$$

as a set of conditions, equivalent to (1.49), that are necessary and sufficient for strong ellipticity.

We show presently that $(2.40)$ with (a) replaced by $\beta_{i}>0$ holds if and only if (3.19) holds. Indeed, suppose (3.19) is true. The first of (3.19) coincides with (2.15) and hence, as shown in Section 2, implies (2.40). Moreover, from the second of (3.19) we draw in particular

$$
K\left(\underset{\sim}{\lambda} ; \zeta_{\sim}\right)>0,
$$

where $\xi_{\rceil}$, introduced in (2.13), is the vertex of $\Lambda$ on the $z_{7}$-axis (see Figure 1). Thus (3.79) necessitates

$$
L_{11}=\lambda_{1}^{-2}\left(\beta_{2}+\beta_{3}\right)>0 \text {, }
$$

which, together with (a) of (2.40), at once requires Note that because of $(3.16),(2.8),(1.4)$, and $(1.14)$, the $L_{i j}$ are
solely functions of the $\lambda_{k}$. 


$$
\begin{aligned}
& -44- \\
& \beta_{i}>0 .
\end{aligned}
$$

Therefore, (3.19) in fact implies (2.40), (3.20), and hence (2.40) with (a) replaced by (3.20).

Conversely, suppose (2.40) and (3.20) hold. Earlier in this section, ${ }^{1}$ we have shown that (2.40) implies the first of (3.19). We now substitute for $w_{i}$ from (2.41) into (3.16) and find - bearing (1.5) in mind - that $K(\lambda ; z)$, defined in (3.18), admits the representation

$$
\begin{aligned}
K(\underset{\sim}{\lambda ; z}) & =\beta_{1}\left(\lambda_{2}^{-1} z_{2}-\lambda_{3}^{-1} z_{3}\right)^{2}+\beta_{2}\left(\lambda_{3}^{-1} z_{3}-\lambda_{1}^{-1} z_{1}\right)^{2}+\beta_{3}\left(\lambda_{1}^{-1} z_{1}-\lambda_{2}^{-1} z_{2}\right)^{2} \\
& +\left[\lambda_{1}^{-2} \beta_{1}+\lambda_{2}^{-2} \beta_{2}+\lambda_{3} \beta_{3}\left(\omega_{3}+2\right)\right] z_{1} z_{2} \\
& +\left[\lambda_{2}^{-2} \beta_{2}+\lambda_{3}^{-2} \beta_{3}+\lambda_{1} \beta_{1}\left(\omega_{1}+2\right)\right] z_{2} z_{3} \\
& +\left[\lambda_{3}^{-2} \beta_{3}+\lambda_{1}^{-2} \beta_{1}+\lambda_{2} \beta_{2}\left(\omega_{2}+2\right)\right] z_{3} z_{1} .
\end{aligned}
$$

Finally, on account of (3.20) and (b) of (2.40), the sum of the first three terms in (3.21) is positive on $\Lambda$ except at the interior point $\stackrel{\circ}{\sim}$ defined by

$$
{\stackrel{\circ}{z_{i}}}_{i}=\lambda_{i}\left(\lambda_{1}+\lambda_{2}+\lambda_{3}\right)^{-1},
$$

whereas the sum of the remaining three terms is positive on $\Lambda$ except at the vertices $\zeta_{i}$. Hence, (3.20) and (b) of (2.40) imply the second of (3.19). This completes the proof of the claim that $(2.40),(3.20)$ hold if and only if (3.19) is true.

See the proof of $(3.7)$. 
The foregoing results entitle us to conclude that a set of conditions necessary and sufficient for the strong ellipticity of the system of partial differential equations (1.10) at a solution $(\underset{\sim}{u}, p)$ and a point $\underset{\sim}{ }$, is given by

$$
\begin{gathered}
\beta_{i}>0, \\
\omega_{i}+2>0, \\
\rho_{1}\left(\omega_{1}-2\right)<\left[\sqrt{\rho_{2}\left(\omega_{2}+2\right)}+\sqrt{\rho_{3}\left(\omega_{3}+2\right)}\right]^{2}, \\
\rho_{2}\left(\omega_{2}-2\right)<\left[\sqrt{\rho_{3}\left(\omega_{3}+2\right)}+\sqrt{\rho_{1}\left(\omega_{1}+2\right)}\right]^{2}, \\
\rho_{3}\left(\omega_{3}-2\right)<\left[\sqrt{\rho_{1}\left(\omega_{1}+2\right)}+\sqrt{\rho_{2}\left(\omega_{2}+2\right)}\right]^{2} .
\end{gathered}
$$

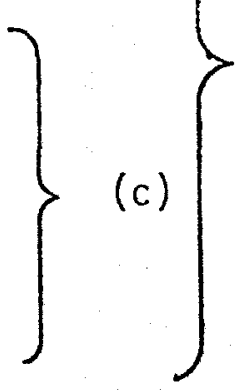

(a)
(b)

(a)
(b)

For convenience, we cite here from $(2.41),(2.42)$ that

$$
\begin{gathered}
\rho_{i}=\beta_{i} / \lambda_{i} \beta_{1} \beta_{2} \beta_{3}, \omega_{i}=w_{i} / \lambda_{j} \beta_{i} \quad \text { (no sums), } \\
\beta_{i}=\hat{W}_{1}\left(I_{1}, I_{2}\right)+\lambda_{i}^{2} \hat{W}_{2}\left(I_{1}, I_{2}\right), \\
W_{i}=\lambda_{i}^{2}\left(I_{1}-\lambda_{i}^{2}\right)_{i}+2 \lambda_{i}^{2}\left(I_{1}^{2}-2 I_{2}-\lambda_{i}^{4}-2 \lambda_{i}^{-2}\right)\left[\hat{W}_{11}\left(I_{1}, I_{2}\right)\right. \\
\left.+2 \lambda_{i}^{2} \hat{W}_{12}\left(I_{1}, I_{2}\right)+\lambda_{i}^{4} \hat{W}_{22}\left(I_{1}, I_{2}\right)\right] \quad \text { (no sum), }
\end{gathered}
$$

where $\lambda_{i}=\lambda_{i} \underset{\sim}{(x)}$ are the local principal stretches associated with $\underset{\sim}{\sim}$ and $I_{\alpha}=I_{\alpha}(\lambda(x))$. With the help of (3.23), the system of inequalities (3.22) is immediately found to be equivalent to the more tractable set

$$
\begin{gathered}
\beta_{i}>0 \\
w_{i}+2 \lambda_{i} \beta_{i}>0 \text { (no sum) },
\end{gathered}
$$




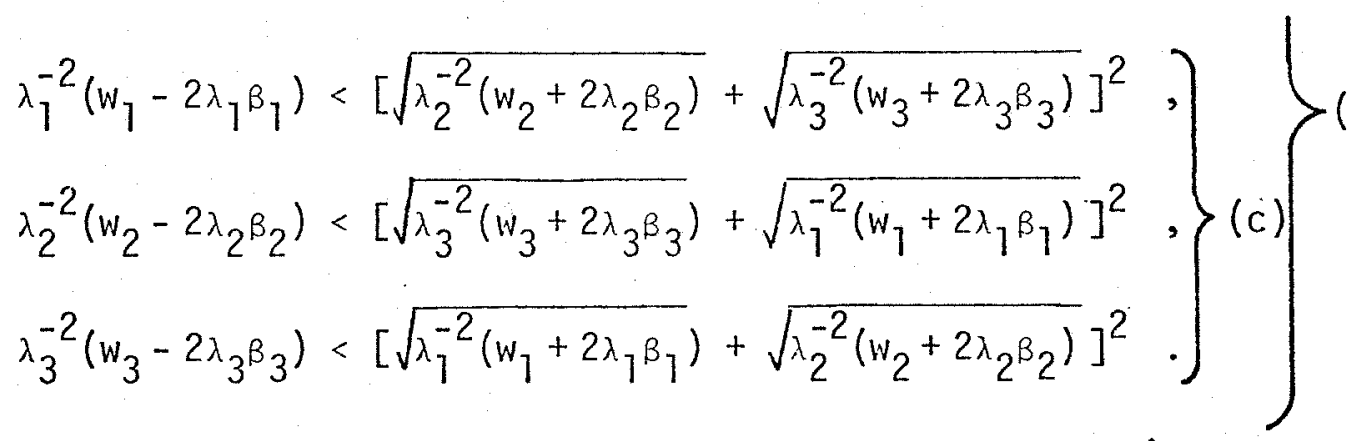

Here $\beta_{j}, w_{j}$ are defined in terms of the derivatives of $\hat{W}$ by $(3.24)$ or alternatively in terms of the derivatives of $\stackrel{*}{W}^{*}$ through (2.53), (2.54).

In order to bring out the invariance of (3.25) under permutations of $\left(\lambda_{1}, \lambda_{2}, \lambda_{3}\right)$, we define functions $x_{j}$ by

$$
\left.\begin{array}{rl}
x_{1}\left(\lambda_{1}, \lambda_{2}\right) & =\beta\left(\lambda_{1}, \lambda_{2}\right), x_{2}\left(\lambda_{1}, \lambda_{2}\right)=w\left(\lambda_{1}, \lambda_{2}\right)+2 \lambda_{1}^{-1} \lambda_{2}^{-1} \beta\left(\lambda_{1}, \lambda_{2}\right), \\
x_{3}\left(\lambda_{1}, \lambda_{2}\right) & =\lambda_{3}^{-2}\left[2 \lambda_{3} \beta\left(\lambda_{1}, \lambda_{2}\right)-w\left(\lambda_{1}, \lambda_{2}\right)\right] \\
& +\left[\sqrt{\lambda_{1}^{-2} x_{2}\left(\lambda_{2}, \lambda_{3}\right)}+\sqrt{\lambda_{2}^{-2} x_{2}\left(\lambda_{3}, \lambda_{1}\right)}\right]^{2}, \lambda_{3}=\left(\lambda_{1} \lambda_{2}\right)^{-1}
\end{array}\right\}
$$

where $\beta$ and $w$ are given by (2.54); thus

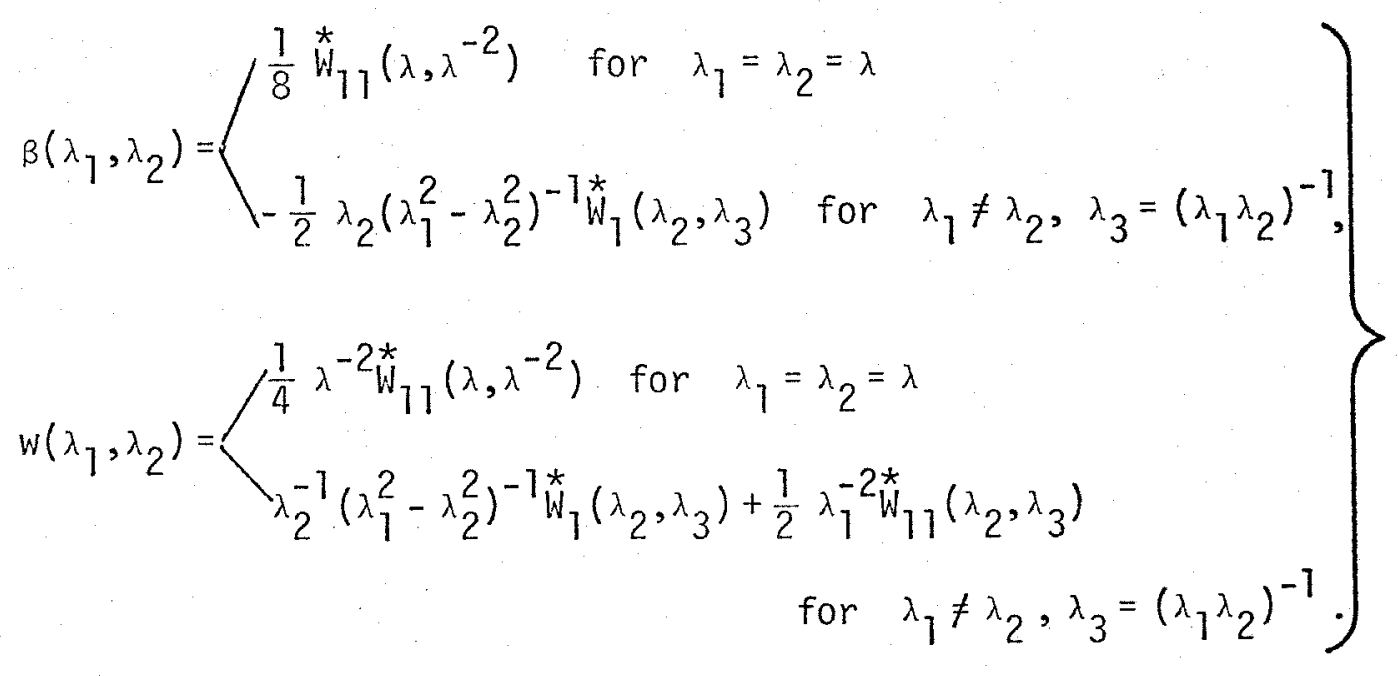

In view of (2.53), the set of inequalities (3.25) may now be written as 


$$
x_{i}\left(\lambda_{1}, \lambda_{2}\right)>0, \quad x_{i}\left(\lambda_{2}, \lambda_{3}\right)>0, \quad x_{i}\left(\lambda_{3}, \lambda_{1}\right)>0
$$

Moreover, from (3.26) and the first two of (2.59), we infer

$$
x_{i}\left(\lambda_{1}, \lambda_{2}\right)=x_{i}\left(\lambda_{2}, \lambda_{1}\right)
$$

By virtue of (3.29), the set of strong ellipticity conditions (3.28)like the system of ordinary ellipticity conditions (2.58) - is fully symmetric with respect to the principal stretches.

We now prove that the conditions for ordinary and strong ellipticity coalesce for an isotropic material of the type under consideration that obeys the Baker-Ericksen inequalities (1.19) and, in addition, has $\underline{\text { a }}$ positive shear modulus at infinitesimal deformations.

Clearly, in the case of distinct $\lambda_{i}$, the inequalities (1.19)in view of (3.24), (1.5) - imply (3.20) and hence the equivalence of (2.40) and (3.22). On the other hand, suppose two of the principal stretches coincide but are different from the third, say $\lambda_{1}=\lambda_{2} \neq \lambda_{3}$. Then (1.19), (3.24), (1.5) give $\beta_{1}=\beta_{2}>0$, and again (2.40) holds if and only if (3.22) is true.

Final7y, suppose all three $\lambda_{i}$ are equal, so that $\lambda_{i}=1$. In this degenerate instance, (2.53), (2.54), and (3.23) yield

$$
\beta_{i}=\frac{1}{2} w_{i}=\frac{1}{8} \stackrel{*}{W}_{11}(1,1), \quad \omega_{i}=2
$$

Thus, conditions (2.40) and (3.22) at present respectively reduce to

$$
\stackrel{\star}{W}_{11}(1,1) \neq 0 \text { and } \stackrel{\star}{W}_{11}(1,1)>0
$$

One easily confirms that if $\mu$ is the shear modulus of the material at infinitesimal deformations, 


$$
\mu=2\left[\hat{W}_{1}(3,3)+\hat{W}_{2}(3,3)\right]=\frac{1}{4} \stackrel{*}{W}_{11}(1,1)
$$

Accordingly, when $\mu>0$, the two conditions in (3.31) are equivalent. This concludes the proof of the preceding claim concerning the equivalence of ordinary and strong ellipticity.

It is of interest to note upon substitution from (3.24), (1.4), (1.5) into (a),(b) of (3.25), that these inequalities hold if and only if

$$
\left.\begin{array}{c}
\hat{W}_{1}+\lambda_{i}^{2} \hat{W}_{2}>0, \\
\hat{W}_{1}+\lambda_{i}^{2} \hat{W}_{2}+2\left(I_{1}-\lambda_{i}^{2}-2 \lambda_{i}^{-1}\right)\left(\hat{W}_{11}+2 \lambda_{i}^{2} \hat{W}_{12}+\lambda_{i}^{4} \hat{W}_{22}\right)>0 \quad \text { (no sum), }
\end{array}\right\}
$$

and consequently one recovers the necessary conditions for strong ellipticity cited by Sawyers [12]. ${ }^{1}$ To see that the additional restrictions (c) of (3.25) are in general independent of (a) and (b), consider the elastic potential defined by

$$
\hat{W}\left(I_{1}, I_{2}\right)=I_{1}+\frac{31}{16} I_{2}-\frac{3}{20} I_{2}^{2}-\frac{597}{80}
$$

and a deformation with local principal stretches

$$
\lambda_{1}=\frac{1}{2}, \quad \lambda_{2}=2, \quad \lambda_{3}=1 .
$$

In this instance, (a) and (b) of (3.25) are satisfied, while the first inequality in (c) is violated.

$\overline{1}$ See inequalities (4.7) of [12]. 
4. Ellipticity conditions for special classes of deformations and materials.

In applying the ellipticity conditions established in the preceding sections to particular classes of deformations and hyperelastic solids, we shall take for granted that the material obeys the Baker-Ericksen inequalities (1.19) and has a positive shear modulus at infinitesimal deformations. Hence, there will be no need to distinguish between the criteria for ordinary and those for strong ellipticity.

We consider first a deformation with local principal stretches

$$
\lambda_{1}=\lambda_{2} \equiv \lambda \quad, \quad \lambda_{3}=\lambda^{-2} .
$$

If $\tau_{i}$ is the principal true stress corresponding to $\lambda_{i}$, induced by this deformation, $(1.17)$ gives

$$
\tau_{1}=\tau_{2} \cdot
$$

Conversely, (4.2), (1.17), and (1.4), (1.5) - because of (1.19) - imply (4.1). Thus, the special deformation at hand is associated with a locally axisymmetric state of true stress. Further, by virtue of the hydrostatic pressure field $\mathrm{p}$, this state may in particular be one of local uniaxial stress, in which case $\tau_{7}=\tau_{2}=0$; alternatively, it may be a state of local equi-biaxial stress with $\tau_{3}=0$.

From (4.1), (2.53), (2.54), and the first two of (2.59) follow

$$
\beta_{1}=\beta_{2}=\beta\left(\lambda^{-2}, \lambda\right) \quad, \quad w_{1}=w_{2}=w\left(\lambda^{-2}, \lambda\right), \quad 2 \lambda_{3} \beta_{3}=w_{3}=w(\lambda, \lambda) .
$$

With the aid of (4.3), the ellipticity conditions (3.25) for a 
deformation locally characterized by (4.1) are readily found to reduce to

$$
\beta_{1}>0, \beta_{3}>0, \quad w_{1}+2 \lambda \beta_{1}>0 .
$$

We show now that the first of these inequalities is automatically satisfied under our present assumptions. Indeed, if $\lambda \neq 1$, the BakerEricksen inequalities (1.19) and the first of the relations (3.24) at once give $\beta_{7}>0$; if $\lambda=1$, in turn, then $\beta_{1}>0$ follows from (3.30) and (3.32) with $\mu>0$. Thus, bearing in mind (3.24), (1.4), and (4.7), one arrives at

$$
\begin{gathered}
\hat{W}_{1}+\lambda^{-4} \hat{W}_{2}>0, \\
\lambda^{4}\left(\hat{W}_{1}+\lambda^{2} \hat{W}_{2}\right)+2\left(\lambda^{3}-1\right)^{2}\left(\hat{W}_{11}+2 \lambda^{2} \hat{W}_{12}+\lambda^{4} \hat{W}_{22}\right)>0,
\end{gathered}
$$

with

$$
\hat{W}_{\alpha}=\hat{W}_{\alpha}\left(I_{1}, I_{2}\right), \hat{W}_{\alpha \beta}=\hat{W}_{\alpha \beta}\left(I_{1}, I_{2}\right), I_{1}=2 \lambda^{2}+\lambda^{-4}, I_{2}=2 \lambda^{-2}+\lambda^{4},
$$

as necessary and sufficient ellipticity conditions in the circumstances under consideration.

It is also useful to cast (4.5), or alternatively the last two inequalities in (4.4), in terms of the partial derivatives of $\stackrel{*}{W}$. We note from the first relation in (a) of (2.49) and the second in (b) that

$$
\stackrel{\star}{W}_{1}\left(\lambda, \lambda^{-2}\right)=0 \quad \forall \lambda>0
$$

Substituting from (3.24), (4.7) into (4.4) and invoking (1.4), one recovers the inequalities (4.8) of Sawyers [12], who observes that the latter are both necessary and sufficient for strong ellipticity so long as the deformation locally conforms to (4.7). 
Thus and by (4.3), (4.1), (3.27), the last two of (4.4) are equivalent to

$$
\stackrel{*}{W}_{11}\left(\lambda, \lambda^{-2}\right)>0, \quad 2 \stackrel{*}{W}_{1}(\lambda, \lambda)+\lambda\left(1+\lambda^{3}\right) \stackrel{*}{W}_{11}(\lambda, \lambda)>0,
$$

so that these two inequalities are also necessary and sufficient ellipticity criteria when the local principal stretches satisfy (4.1).

Consider next a deformation corresponding to a state of local plane strain with principal stretches

$$
\lambda_{1} \equiv \lambda, \lambda_{2}=\lambda^{-1}, \quad \lambda_{3}=1 .
$$

In this instance our assumptions (1.19) and $\mu>0$ are easily seen to imply $\beta_{i}>0$. In fact, if $\lambda \neq 1,(1.19)$ and (3.24) immediately yield $\beta_{j}>0$, whereas for $\lambda=1$ the $\beta_{j}$ are positive as a consequence of (3.30) and (3.32). Thus, for local plane strain, the only ellipticity conditions to survive are (b) and (c) in (3.25).

Abeyaratne [6] deduced necessary and sufficient conditions of ordinary ellipticity for a deformation of global plane strain with a displacement field obeying

$$
u_{\alpha, 3}=0, \quad u_{3}=0 .
$$

Such a deformation gives rise to (4.9) throughout the body. The conditions of ordinary ellipticity arrived at in [6] are

$$
W^{\prime}(I) \neq 0, \quad 1+2(I-2)\left[W^{\prime \prime}(I) / W^{\prime}(I)\right]>0,
$$

See inequalities (3.21) in [6]. 
where

$$
I=I_{1}-1=I_{2}-1=\lambda^{2}+\lambda^{-2}, \quad W(I)=\hat{W}(I+1, I+1)
$$

We now verify that (4.11) is at present implied by (b) and (c) in (3.25), as must be the case when the Baker-Ericksen inequalities (1.19) are assumed to hold and $\mu>0$. Indeed, one gathers from (3.24), (4.12), and (4.9) that

$$
W^{\prime}(I)=\beta_{3}, \quad W^{\prime}(I)+2(I-2) W^{\prime \prime}(I)=\lambda^{2}\left(\lambda^{2}+1\right)^{-2}\left(W_{3}+2 \lambda_{3} B_{3}\right) .
$$

But $w_{3}+2 \lambda_{3} \beta_{3}>0$ according to (b) in (3.25), white $\beta_{3}>0$ under the assumptions just mentioned, whence (4.11) follows.'

The necessary and sufficient ellipticity conditions appropriate to a local state of plane strain governed by (4.9) are in general far more restrictive than (4.11) since the former involve all three inequalities in (b) of (3.25), as well as the three inequalities in (c). These additional restrictions stem from the fact that the local hypothesis (4.9) admits a larger class of deformations than does the glabal requirement (4.10); furthermore, (4.11) - with (1.19) in force - preclude potential discontinuities in the relevant normal derivatives of the displacements and pressure merely across cylindrical surfaces with generators parallel to the principal direction of the deformation tensor $\underset{\sim}{\mathcal{C}}$ that is associated with $\lambda_{3}=1$.

\footnotetext{
It should be noted that (4.11), as expected, is also implied by the conditions of ordinary ellipticity (2.40) even if the Baker-Ericksen inequalities fail to hold or if $\mu \leq 0$.
} 
We proceed now to special classes of materials and in this connection consider first elastic potentials that depend on a single deformation invariant. Suppose

$$
\hat{W}\left(I_{1}, I_{2}\right)=W\left(I_{1}\right)
$$

We show presently that in this event, (a) and (b) imply (c) in the ellipticity conditions (3.25). ${ }^{1}$ From $(4.14)$ and $(1.4),(1.5),(3.24)$ we draw

$$
\left.\begin{array}{c}
\beta_{i}=W^{\prime}\left(I_{1}\right), \\
\lambda_{j}^{-2}\left(W_{i} \pm 2 \lambda_{i} \beta_{j}\right)=\left(I_{1}-\lambda_{i}^{2} \pm 2 \lambda_{i}^{-7}\right)\left[W^{\prime}\left(I_{1}\right)+2\left(I_{1}-\lambda_{i}^{2} \mp 2 \lambda_{i}^{-7}\right) W^{\prime \prime}\left(I_{1}\right)\right] \\
\text { (no sum) }
\end{array}\right\}(4.15)
$$

Assume now that (a) and (b) in (3.25) hold true, or equivalently

$$
W^{\prime}\left(I_{7}\right)>0, \quad W^{\prime}\left(I_{1}\right)+2\left(I_{1}-\lambda_{j}^{2}-2 \lambda_{i}^{-1}\right) W^{\prime \prime}\left(I_{1}\right)>0
$$

With a view towards inferring the first inequality in (c) of (3.25), we note that the latter can be written as

$$
S<T^{1 / 2}
$$

provided

$$
\begin{aligned}
& S=\lambda_{1}^{-2}\left(w_{1}-2 \lambda_{1} \beta_{1}\right)-\lambda_{2}^{-2}\left(w_{2}+2 \lambda_{2} \beta_{2}\right)-\lambda_{3}^{-2}\left(w_{3}+2 \lambda_{3} \beta_{3}\right), \\
& T=4 \lambda_{1}^{2}\left(w_{2}+2 \lambda_{2} \beta_{2}\right)\left(w_{3}+2 \lambda_{3} \beta_{3}\right),
\end{aligned}
$$

Clearly, $\mathrm{T}>0$ under our current hypotheses.

Tone may show similarly that (a) and (b) imply (c) in the ordinary ellipticity conditions (2.40) whenever (4.14) holds. 
On account of (4.18) and (4.15), (1.4), (1.5),

$$
\begin{aligned}
& S=-2\left(\lambda_{1}+\lambda_{2}\right)\left(\lambda_{1}+\lambda_{3}\right)\left[W^{\prime}\left(I_{1}\right)+2\left(\lambda_{1}-\lambda_{2}\right)\left(\lambda_{1}-\lambda_{3}\right) W^{\prime \prime}\left(I_{1}\right)\right], \\
& T=S^{2}+U
\end{aligned}
$$

where

$$
U=8\left(\lambda_{1}+\lambda_{2}\right)^{2}\left(\lambda_{1}+\lambda_{3}\right)^{2}\left(\lambda_{2}-\lambda_{3}\right)^{2} W^{\prime}\left(I_{1}\right) W^{\prime \prime}\left(I_{1}\right) .
$$

In order to establish (4.17), it is convenient to observe that

$$
\text { either } \lambda_{2}=\lambda_{3} \text { or } \lambda_{2} \neq \lambda_{3}, W^{\prime \prime}\left(I_{7}\right) \leq 0 \text { or } \lambda_{2} \neq \lambda_{3}, W^{\prime \prime}\left(I_{7}\right)>0 \text {. }
$$

If the first of these three eventualities holds, (4.16), (4.19) imply

$$
S=-2\left(\lambda_{1}+\lambda_{2}\right)^{2}\left[W^{\prime}\left(I_{1}\right)+2\left(\lambda_{1}-\lambda_{2}\right)^{2} W^{\prime \prime}\left(I_{1}\right)\right]<0,
$$

and hence (4.17) follows. On the other hand, the second alternative in (4.21) justifies

$$
2\left(\lambda_{1}-\lambda_{2}\right)\left(\lambda_{1}-\lambda_{3}\right) W^{\prime \prime}\left(I_{1}\right) \geq \min \left[2\left(\lambda_{1}-\lambda_{2}\right)^{2} W^{\prime \prime}\left(I_{1}\right), 2\left(\lambda_{1}-\lambda_{3}\right)^{2} W^{\prime \prime}\left(I_{1}\right)\right],
$$

so that (4.16), (4.19) again yield $S<0$ and thus (4.17). Finally, the last alternative in (4.21), together with (4.20), (4.19), and the first of (4.16), gives $U>0$ and $T>S^{2}$, which confirms (4.17) in this case also. Strictly parallel arguments enable one to infer the remaining two inequalities in (c) of (3.25).

As is now clear, (4.16) constitutes necessary and sufficient ellipticity conditions when the elastic potential obeys (4.14). Moreover, the Baker-Ericksen inequalities and $\mu>0$ imply the first of the two inequalities (4.16.) 
If, instead of (4.14),

$$
\hat{W}\left(I_{1}, I_{2}\right)=W\left(I_{2}\right) \text {, }
$$

one finds similarly that (a), (b) once again imply (c) in (3.25) and is led to

$$
W^{\prime}\left(I_{2}\right)>0, \quad W^{\prime}\left(I_{2}\right)+2\left(I_{2}-\lambda_{i}^{-2}-2 \lambda_{j}\right) W^{\prime \prime}\left(I_{2}\right)>0,
$$

as necessary and sufficient ellipticity conditions.

It should be mentioned that the ellipticity conditions (4.16) and (4.23), appropriate to (4.14) and (4.22), respectively, recover earlier results reported by Sawyers [12]. ${ }^{1}$

Consider now the special case of a Mooney-Rivlin material, for which

$$
\hat{W}\left(I_{1}, I_{2}\right)=c_{1}\left(I_{1}-3\right)+c_{2}\left(I_{2}-3\right) \text {. }
$$

The Baker-Ericksen inequalities (1.19) necessitate

$$
c_{\alpha} \geq 0, \quad \frac{1}{2} \mu \equiv c_{1}+c_{2}>0
$$

and with (4.25) in force, the ellipticity conditions (3.25) are found to be automatically satisfied for all principal stretches. Thus, for a Mooney-Rivlin material that obeys the Baker-Ericksen inequalities, ellipticity obtains at al1 deformations.

Finally, as an illustrative example, we apply the ellipticity conditions established in this paper to a class of hypothetical materials

See Section 5 of [12]. 
introduced by ogden [13]. The associated mechanical response is governed by an elastic potential of the form

$$
\stackrel{*}{W}\left(\lambda_{1}, \lambda_{2}\right)=\frac{c}{\alpha}\left(\lambda_{1}^{\alpha}+\lambda_{2}^{\alpha}+\lambda_{3}^{\alpha}-3\right), \quad \lambda_{3}=\left(\lambda_{1} \lambda_{2}\right)^{-1}, \quad c>0, \alpha>0 .
$$

According to (3.27) and (4.7) one has at present

$$
\begin{aligned}
& \beta\left(\lambda_{1}, \lambda_{2}\right)=\left\{\begin{array}{l}
\frac{\alpha C}{4} \lambda^{\alpha-2} \quad \text { for } \lambda_{1}=\lambda_{2}=\lambda . \\
\frac{c}{2}\left(\lambda_{1}^{\alpha}-\lambda_{2}^{\alpha}\right) /\left(\lambda_{1}^{2}-\lambda_{2}^{2}\right) \text { for } \lambda_{1} \neq \lambda_{2}
\end{array},\right. \\
& \left(\lambda_{1} \lambda_{2}\right)^{2}\left[w\left(\lambda_{1}, \lambda_{2}\right)+2\left(\lambda_{1} \lambda_{2}\right)^{-1} B\left(\lambda_{1}, \lambda_{2}\right)\right] \\
& =\frac{c}{2} \lambda_{2}^{\alpha}(1+\delta)^{-1}\left[(\alpha-1)\left(1+\delta^{\alpha+1}\right)+(\alpha+1)\left(\delta+\delta^{\alpha}\right)\right] \text {, } \\
& \left(\lambda_{1} \lambda_{2}\right)^{2}\left[W\left(\lambda_{1}, \lambda_{2}\right)-2\left(\lambda_{1} \lambda_{2}\right)^{-1} B\left(\lambda_{1}, \lambda_{2}\right)\right] \\
& =\left(\begin{array}{l}
0 \text { for } \lambda_{1}=\lambda_{2} \\
\frac{c}{2}\left(\lambda_{1}^{\alpha}+\lambda_{2}^{\alpha}\right)\left[\alpha-\frac{(\delta+1)\left(\delta^{\alpha}-1\right)}{(\delta-1)\left(\delta^{\alpha}+1\right)}\right] \text { for } \lambda_{1} \neq \lambda_{2},
\end{array}\right)
\end{aligned}
$$

where $\delta=\lambda_{1} / \lambda_{2}$. With the aid of (4.27) and by recourse to (2.53), one can show that

$$
\beta_{i}>0,
$$

$$
\left.\begin{array}{l}
\lambda_{i}^{-2}\left(w_{i}+2 \lambda_{j} \beta_{i}\right)>\frac{c}{2}\left(\lambda_{1}^{\alpha}+\lambda_{2}^{\alpha}+\lambda_{3}^{\alpha}-\lambda_{j}^{\alpha}\right)(\alpha-1) \quad \text { (no sum) }, \\
\lambda_{i}^{-2}\left(w_{i}-2 \lambda_{i} \beta_{i}\right) \leq \frac{c}{2}\left(\lambda_{1}^{\alpha}+\lambda_{2}^{\alpha}+\lambda_{3}^{\alpha}-\lambda_{i}^{\alpha}\right) \max [0, \alpha-1] \quad \text { (no sum). }
\end{array}\right\}
$$

The first of (4.28) implies the Baker-Ericksen inequalities (1.19), and 
(4.26) - together with (3.32)-gives $\mu=c \alpha / 2>0$; further, (a) in (3.25) is satisfied for all $\lambda_{i}$ and all $\alpha>0$. Moreover, when $\alpha \geq 1$, we easily see that (b) and (c) in (3.25) also hold for all $\lambda_{i}$. In contrast, as may be shown by means of the second of (4.27) and the last of (4.28), for $0<\alpha<1,(b)$ and (c) hold if and only if

$$
\varepsilon<\lambda_{j} / \lambda_{j}<\varepsilon^{-1}
$$

provided $\varepsilon=\varepsilon(\alpha)$ is the unique solution of the equation

$$
(1+\alpha)\left(\varepsilon+\varepsilon^{\alpha}\right)=(1-\alpha)\left(1+\varepsilon^{\alpha+1}\right), \quad 0<\varepsilon<1 .
$$

Therefore, for an elastic potential obeying (4.26) with $\alpha \geq 1$, ellipticity prevails at all deformations, whereas if $0<\alpha<1$, the inequalities (4.29) are necessary and sufficient for ellipticity. ${ }^{l}$

The inequalities (4.29), which are contingent upon $0<\alpha<1$, describe the interior $\Omega$ of a convex hexagonal pyramid in the space of the principal stretches, ${ }^{2}$ the origin of which coincides with the vertex of this pyramid. The intersection $\Sigma$ of $\Omega$ with the "isochoric surface" $\lambda_{1} \lambda_{2} \lambda_{3}=1$ represents the ellipticity domain in this instance.

Evidently, (4.30) can be solved explicitiy if $\alpha=\frac{1}{2}$. In this special case, the ellipticity conditions (4.29), upon elimination of $\lambda_{3}$, may be written as

$$
\varepsilon<\lambda_{1} \lambda_{\overline{2}}^{-1}<\varepsilon^{-1}, \quad \varepsilon<\lambda_{1} \lambda_{2}^{2}<\varepsilon^{-1}, \quad \varepsilon<\lambda_{1}^{2} \lambda_{2}<\varepsilon^{-1}, \quad \varepsilon=7-4 \sqrt{3} \doteq 0.0718 .
$$

Ogden [13], on the basis of tests performed on vulcanized natural rubber, suggests the choice $\alpha=1.2$ and $c=6.8 \mathrm{~kg} / \mathrm{cm}^{2}$.

${ }^{2}$ See the interpretation of inequalities (3.1) of [1]. 
Conditions (4.31) define the open region $\Sigma_{12}$ in the $\left(\lambda_{1}, \lambda_{2}\right)$-plane depicted as the shaded area in Figure 2. This region is the projection onto the $\left(\lambda_{1}, \lambda_{2}\right)$-plane of the ellipticity domain $\Sigma$; the projections $\Sigma_{23}$ and $\Sigma_{31}$ of $\Sigma$ onto the other two principal-stretch planes are once again represented by the shaded region in Figure 2 , provided $\left(\lambda_{1}, \lambda_{2}\right)$ in this diagram is replaced by $\left(\lambda_{2}, \lambda_{3}\right)$ or by $\left(\lambda_{3}, \lambda_{7}\right)$.

It is of interest to examine the response, to certain basic homogeneous deformations, of the material characterized by the elastic potential (4.26). For a pure homogeneous, volume-preserving deformation of the form

$$
y_{i}=\lambda_{i} x_{i} \quad(\text { no sum }), \quad \lambda_{7} \lambda_{2} \lambda_{3}=1,
$$

one gathers from (1.7), (1.16), the first of (2.46), and (4.26) that

$$
\tau_{i j}=\sigma_{i j}=0 \quad(i \neq j),
$$

while

$$
\lambda_{j}^{\sigma}{ }_{i j}=\tau_{i j}, \tau_{\beta B}-\tau_{33}=\lambda_{\beta}{\underset{W}{W}}_{\beta}\left(\lambda_{1}, \lambda_{2}\right)=c\left(\lambda_{\beta}^{\alpha}-\lambda_{3}^{\alpha}\right) \text { (no sum over } i \text { or } \beta \text { ). }
$$

In particular, for a pure homogeneous deformation of (i) uniaxial stress or (ii) equi-biaxial stress or ( $i i i)$ plane-strain uniaxial stress ("pure shear"), (4.33) yields respectively:

(i) $\tau_{11}=\tau_{22}=0, \lambda \sigma_{33}(\lambda)=\tau_{33}(\lambda)=c\left(\lambda^{\alpha}-\lambda^{-\alpha / 2}\right), \lambda_{3}=\lambda, \lambda_{1}=\lambda_{2}=\lambda^{-1 / 2}$;

(ii) ${ }_{\tau_{33}}=0, \quad \lambda \sigma_{22}(\lambda)=\tau_{22}(\lambda)=c\left(\lambda^{\alpha}-\lambda^{-2 \alpha}\right), \quad \lambda_{1}=\lambda_{2}=\lambda, \lambda_{3}=\lambda^{-2}$;

(iii) $\tau_{22}=0, \quad \lambda_{11}(\lambda)={ }_{11}(\lambda)=c\left(\lambda^{\alpha}-\lambda^{-\alpha}\right), \quad \lambda_{1}=\lambda_{2}^{-1}=\lambda, \lambda_{3}=1$. 
Next, consider a deformation of simple shear given by

$$
y_{1}=x_{1}+k x_{2}, \quad y_{2}=x_{2}, \quad y_{3}=x_{3},
$$

where $k$ is the amount of shear. The corresponding principal stretches obey

$$
\lambda_{1}=\lambda_{2}^{-1}=\frac{1}{2}\left(k+\sqrt{k^{2}+4}\right), \quad \lambda_{3}=1, \quad k=\lambda_{1}-\lambda_{1}^{-1},
$$

and the in-plane true shear stress $\tau_{12}$ here is found to satisfy

$$
{ }^{\tau} 12=\tau(\lambda)=c\left(\lambda^{\alpha}-\lambda^{-\alpha}\right) /\left(\lambda+\lambda^{-1}\right), \quad \lambda=\lambda_{1} .
$$

When $\alpha \geq 1$, the normal stresses $\sigma_{33}(\lambda),{ }^{\tau}{ }_{33}(\lambda)$ and $\sigma_{22}(\lambda),{ }_{22}(\lambda)$, as well as $\sigma_{11}(\lambda), \tau 11(\lambda)$, arising in the pure homogeneous deformations (4.34), are monotone increasing functions of $\lambda$ for $0<\lambda<\infty$. When $0<a<1$, on the other hand, this monotonicity property is retained by the true stresses $\tau_{33}(\lambda), \tau_{22}(\lambda), \tau_{11}(\lambda)$, but the associated nominal stresses $\sigma_{33}(\lambda), \sigma_{22}(\lambda), \sigma_{11}(\lambda)$ are steadily increasing merely for $0<\lambda<\lambda_{\star}$, possess a maximum at $\lambda=\lambda_{\star}$, and monotonically decrease to zero on $\lambda_{*}<\lambda<\infty$, where from (4.34),

$$
\begin{aligned}
& \text { (i) } \lambda_{*}=[(2+\alpha) / 2(1-\alpha)]^{2 / 3 \alpha}>1, \\
& \text { (ii) } \lambda_{*}=[(1+2 \alpha) /(1-\alpha)]^{1 / 3 \alpha}>1, \\
& \text { (iii) } \lambda_{*}=[(1+\alpha) /(1-\alpha)]^{1 / 2 \alpha}>1,
\end{aligned}
$$

respectively. The graphs of $\sigma_{33}(\lambda)$ and $\tau_{33}(\lambda)$ appropriate to case (i) of uniaxial stress are shown ${ }^{1}$ in Figure 3 for the three values $\alpha=3 / 2$,

In the interest of clarity, the curves for $\tau_{33}(\lambda)$ have been omitted for $0<\lambda<1$. 
$\alpha=1, \alpha=1 / 2$. The graphs of $\sigma_{22}(\lambda), \tau_{22}(\lambda)$ and $\sigma_{17}(\lambda), \tau_{11}(\lambda)$, corresponding to the cases ( $i i)$ and $(i j i)$, are qualitatively quite similar.

As far as the simple-shear deformation (4.35) is concerned, (4.37) reveals $\tau(\lambda)$ to be steadily increasing over the entire range $0<\lambda<\infty$, provided $\alpha \geq 1$. When $0<\alpha<1$, however, $\tau(\lambda)$ increases monotonically only for $\varepsilon^{1 / 2}<\lambda<\varepsilon^{-1 / 2}$, with $\varepsilon=\varepsilon(\alpha)$ given by the root of (4.30); for this range of the parameter $\alpha, \tau(\lambda)$ has a minimum at $\lambda=\varepsilon^{1 / 2}$, a maximum at $\lambda=\varepsilon^{-1 / 2}$, and is strictly decreasing for $0<\lambda<\varepsilon^{1 / 2}$ and $\varepsilon^{-1 / 2}<\lambda<\infty$. The graphs of $\tau(\lambda)$ corresponding to $\alpha=3 / 2, \alpha=1$, and $\alpha=1 / 2$ are displayed in Figure 4 .

It is evident from the foregoing discussion of the three pure homogeneous deformations introduced in (4.34) that when $0<\alpha<1$, the nominal normal stresses singled out there are no longer invertible functions of the appropriate principal stretch $\lambda$ over the range $1<\lambda<\infty$, which corresponds to an elongation. No such loss of invertibility is encountered in contraction, i.e., for $0<\lambda<1$. Furthermore, ellipticity is lost when $0<\alpha<1$ in both extension and contraction, and as illustrated by Figure 3 - which pertains to uniaxial stress - the maximum of $\sigma_{33}(\lambda)$ occurs in the interior of the range of ellipticity. ${ }^{1}$

As regards the behavior in simple shear of the special material under discussion, we nōte that here, for $0<\alpha<1$, the range of

In case ( $i i)$, which refers to equi-biaxial stress, invertibility of $\sigma_{22}(\lambda)$ is lost once again only in extension, but this loss occurs outside the range of ellipticity for $0<\alpha<\alpha_{*} \doteq 0.714$ and falls inside this range for $\alpha_{\star}<\alpha<1$. 
ellipticity

$$
[\varepsilon(\alpha)]^{1 / 2}<\lambda<[\varepsilon(\alpha)]^{-1 / 2}
$$

precisely coincides with the range of $\lambda$ over which the shear-response function $\tau(\lambda)$ is monotone increasing, and the two extrema of $\tau(\lambda)$ occur at the endpoints of this range (see Figure 4).

It is now also apparent that for the material characterized by (4.26), ellipticity prevails at a local state of plane strain with $\lambda_{1}=\lambda_{2}^{-1}=\stackrel{\circ}{\lambda}, \lambda_{3}=1$ if and only if the shear-response function $\tau(\lambda)$ has a positive slope at $\lambda=\stackrel{\circ}{\lambda}$. This conclusion is in complete accord with a result previously arrived at by Abeyaratne [6]. A related conclusion had been reached still earlier by Knowles [4], who found that the displacement equations of equilibrium appropriate to global anti-plane shear of a class of incompressible hyperelastic materials are locally elliptic at a solution if and only if the response curve for simple shear has a positive slope at an amount of shear equal to the magnitude of the local displacement gradient.

The preceding observations suggest that a loss of ellipticity in finite elastostatics always entails some loss of invertibility of the underlying stress-deformation relations. A physical interpretation along these lines of the three-dimensional ellipticity conditions established in this paper has so far eluded our efforts. 


\section{References}

[1] J. K. Knowles and Eli Sternberg, On the ellipticity of the equations of nonlinear elastostatics for a special material, Journal of Elasticity, $\underline{5}$ (1975), 3-4, 341 .

[2] J. K. Knowles and Eli Sternberg, On the failure of ellipticity of the equations for finite elastostatic plane strain, Archive for Rational Mechanics and Analysis, 63 (1977), 4, 321.

[3] J. K. Knowles and Eli Sternberg, On the failure of ellipticity and the emergence of discontinuous deformation gradients in plane finite elastostatics, Journal of Elasticicy, $\underline{8}$ (1978), 4, 329.

[4] J. K. Knowles, on the dissipation associated with equilibrium shocks in finite elasticity, Journal of Elasticity, 9 (1979), 2, 131.

[5] J. K. Knowles, The finite anti-plane shear field near the tip of a crack for a class of incompressible elastic solids, International Journal of Fracture, 13 (1977), 5, 611 .

[6] R. C. Abeyaratne, Discontinuous deformation gradients in plane finite elastostatics of incompressible materials, Journal of Elasticity, 10 (1980), 3, 255.

[7] Eli Sternberg, On singular problems in linearized and finite elastostatics in Proceedings, 15th International Congress of Theoretical and Applied Mechanics, Toronto, North-Holland, Amsterdam, 1980.

[8] R. C. Abeyaratne, Discontinuous deformation gradients away from the tip of a crack in anti-plane shear, Journal of Elasticity, 11 
(1981), 4, 373 .

[9] J.W. Rudnicki and J. R. Rice, Conditions for the localization of deformation in pressure-sensitive dilatant materials, Journal of the Mechanics and Physics of Solids, 23 (1975), 6, 371.

[10] R. Hill and J. W. Hutchinson, Bifurcation phenomena in the plane tension test, Journal of the Mechanics and Physics of Solids, 23 (1975), 4/5, 239.

[11] K. N. Sawyers and R. S. Rivlin, Instability of an elastic material, International Journal of Solids and Structures, $\underline{9}$ (1973), 5, 607.

[12] K. N. Sawyers, Material stability and bifurcation in finite elasticity in Finite Elasticity, Applied Mechanics Symposia Series, Vol. 27, ASME, New York, 1977.

[13] R. W. Ogden, Large deformation isotropic elasticity - on the correlation of theory and experiment for incompressible rubberlike solids, Proceedings, Royal Society of London, A 326 (1972), 565.

[14] L. E. Dickson, Theory of Equations, Wiley, New York, 1939. 


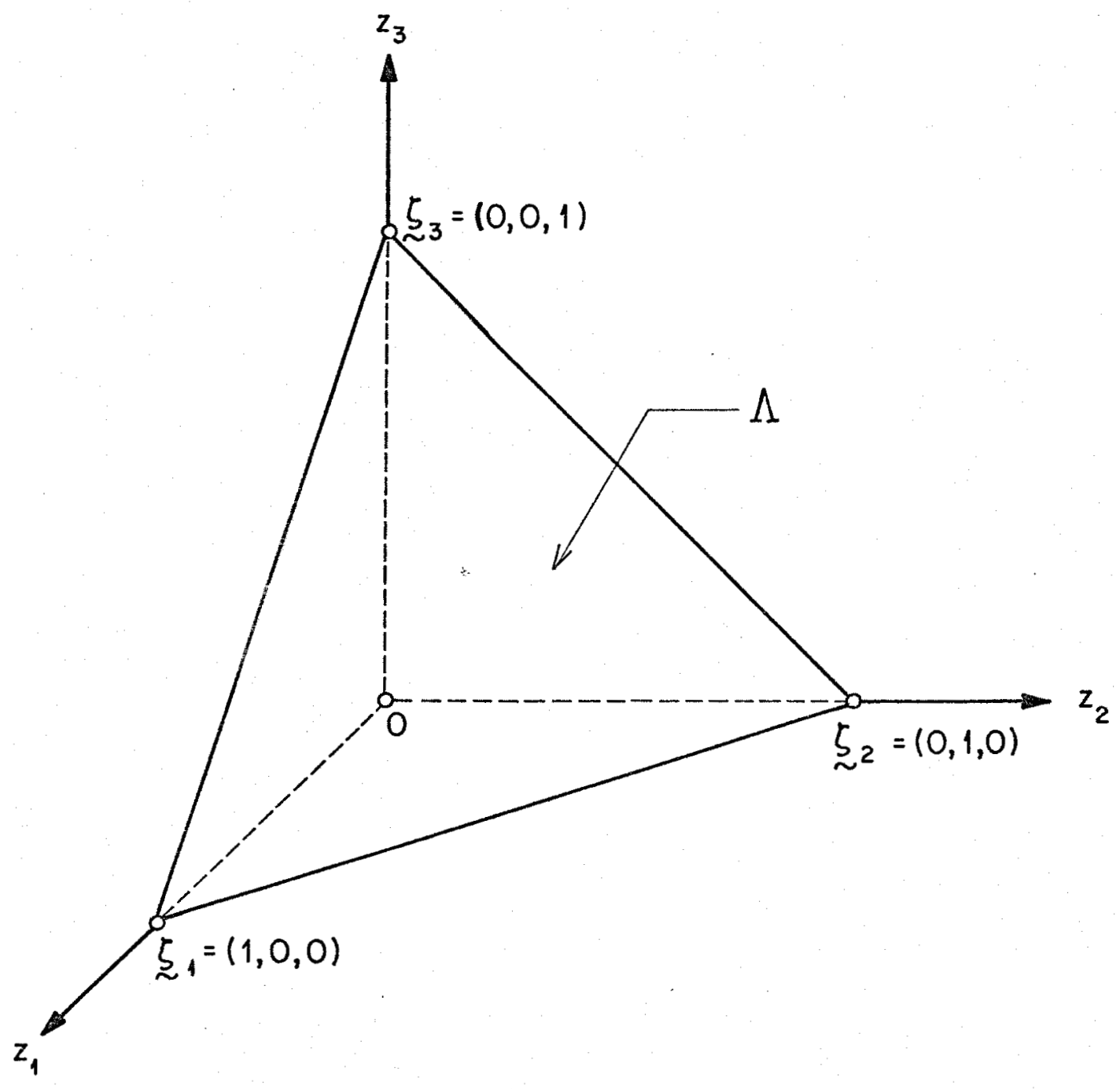

FIGURE 1. THE PLANE REGION $\Lambda$ IN $\underset{\sim}{z}$-SPACE. 


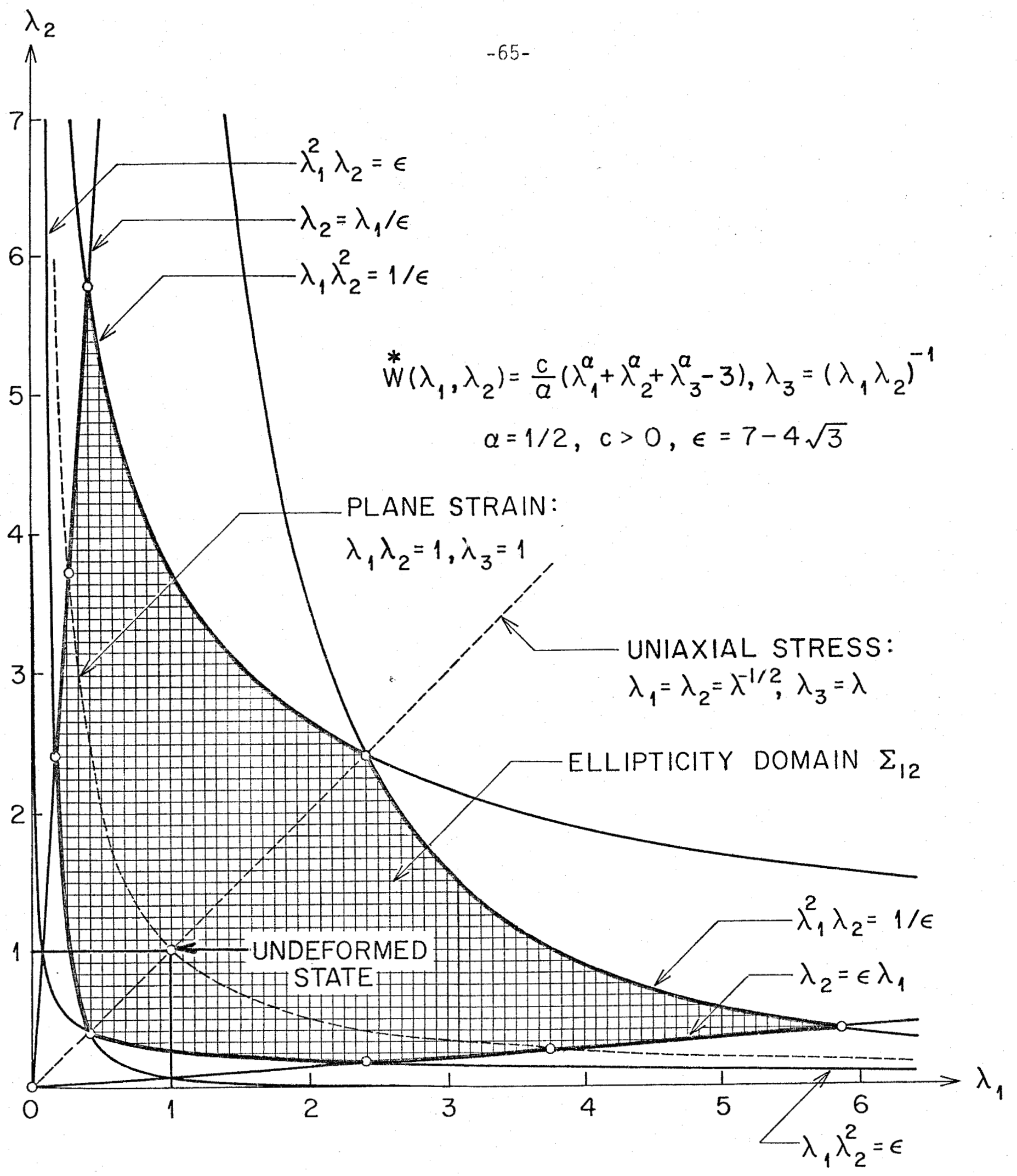

FIGURE 2. ELLIPTICITY DOMAIN IN THE $\left(\lambda_{1}, \lambda_{2}\right)$-PLANE FOR THE SPECIAL MATERIAL. 


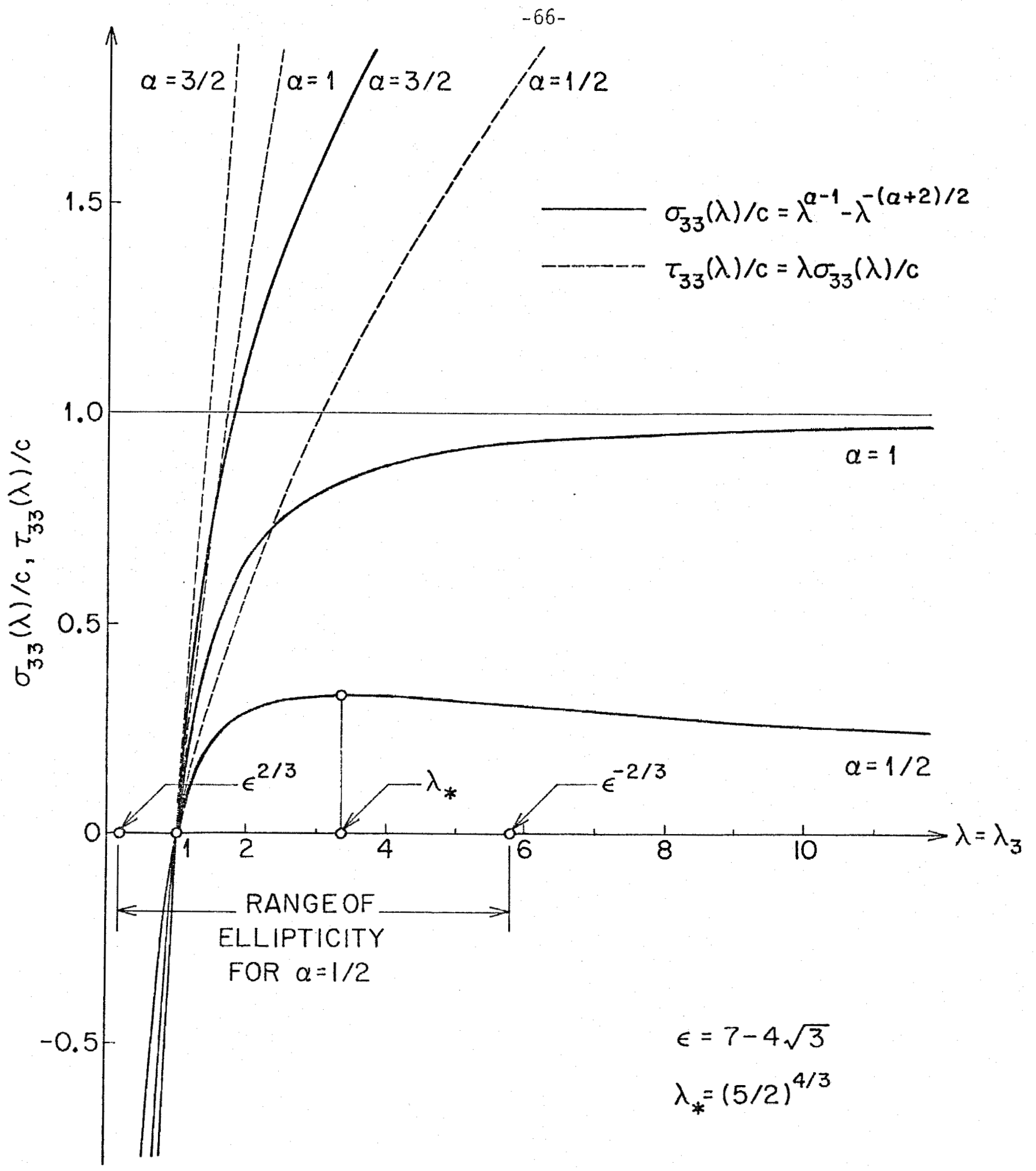

FIGURE 3. BEHAVIOR OF THE SPECIAL MATERIAL UNDER UNIAXIAL STRESS. 
$\tau(\lambda) / c$

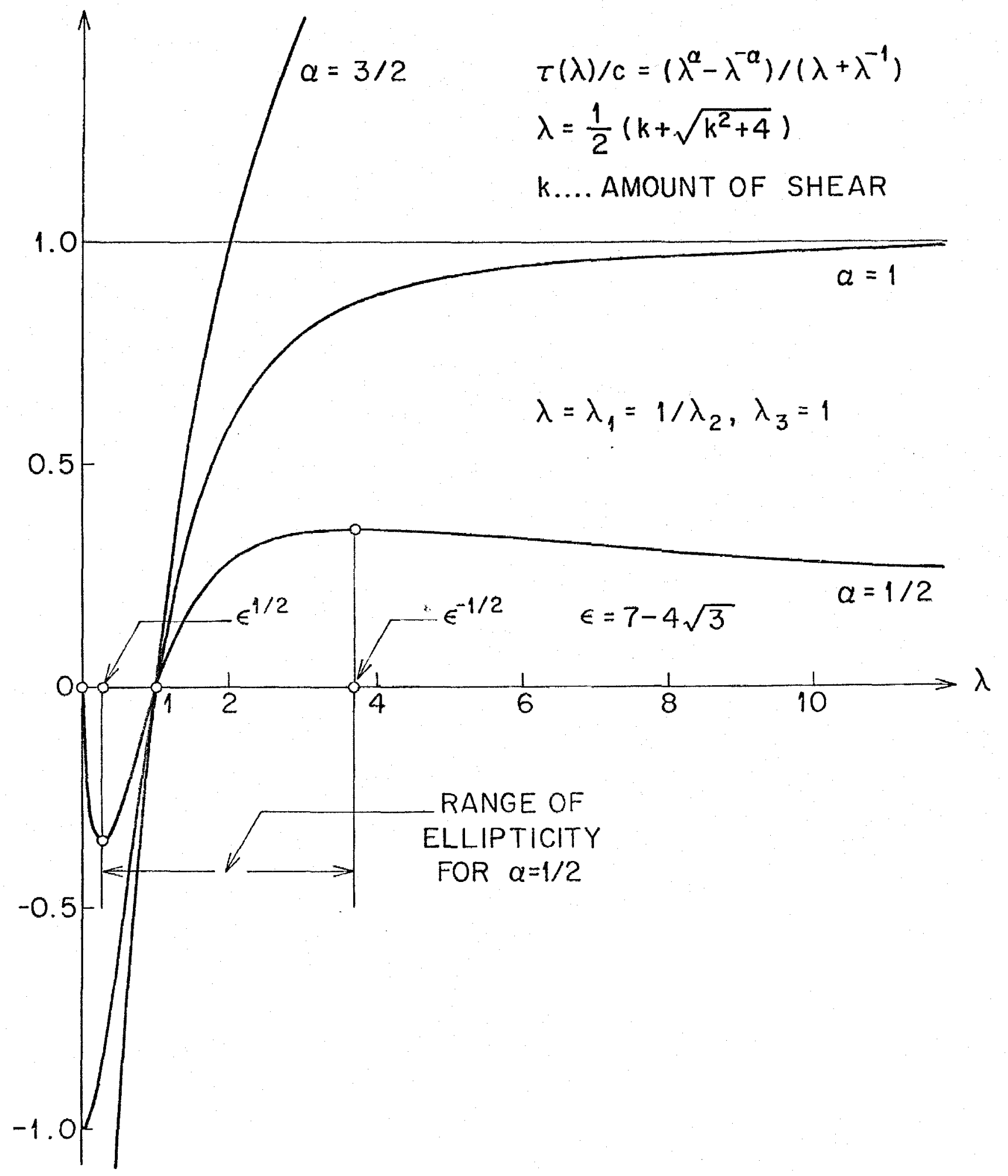

FIGURE 4. RESPONSE OF THE SPECIAL MATERIAL TO SIMPLE SHEAR. 